4-1-1993

\title{
Contribution of High-Order Rainbows to the Scattering of a Gaussian Laser Beam by a Spherical Particle
}

James A. Lock

Cleveland State University, j.lock@csuohio.edu

Follow this and additional works at: https://engagedscholarship.csuohio.edu/sciphysics_facpub

Part of the Physics Commons

How does access to this work benefit you? Let us know!

\section{Publisher's Statement}

This paper was published in Journal of the Optical Society of America A: Optics Image Science and Vision and is made available as an electronic reprint with the permission of OSA. The paper can be found at the following URL on the OSA website: http://www.opticsinfobase.org/josaa/ abstract.cfm?URI=josaa-10-4-693. Systematic or multiple reproduction or distribution to multiple locations via electronic or other means is prohibited and is subject to penalties under law.

\section{Original Citation}

Lock, James A. "Contribution of High-Order Rainbows to the Scattering of a Gaussian Laser Beam by a Spherical Particle." Journal of the Optical Society of America A: Optics Image Science and Vision 10 (1993): 693-706.

\section{Repository Citation}

Lock, James A., "Contribution of High-Order Rainbows to the Scattering of a Gaussian Laser Beam by a Spherical Particle" (1993). Physics Faculty Publications. 4.

https://engagedscholarship.csuohio.edu/sciphysics_facpub/4

This Article is brought to you for free and open access by the Physics Department at EngagedScholarship@CSU. It has been accepted for inclusion in Physics Faculty Publications by an authorized administrator of EngagedScholarship@CSU. For more information, please contact library.es@csuohio.edu. 


\title{
Contribution of high-order rainbows to the scattering of a Gaussian laser beam by a spherical particle
}

\author{
James A. Lock \\ Department of Physics, Cleveland State University, Cleveland, Ohio 44115
}

Received June 18, 1992; revised manuscript received October 5, 1992; accepted October 5, 1992

\begin{abstract}
I review the theory of the scattering of a Gaussian laser beam by a dielectric spherical particle and give the details for constructing a computer program to implement the theory. Computational results indicate that if the width of the laser beam is much less than the diameter of the particle and if the axis of the beam is incident near the edge of the particle, the fifth-, sixth-, and ninth-order rainbows should be evident in the far-field scattered intensity. I performed an experiment that yielded tentative evidence for the presence of the sixthorder rainbow.
\end{abstract}

\section{INTRODUCTION}

In the operation of a number of particle-sizing instruments the diameter of a small liquid droplet is determined, as it passes through the focal waist of a focused laser beam, from the various details of its far-field lightscattering signature. ${ }^{1-6}$ For such instruments the diameter of the droplet is often comparable with the width of the laser beam focal waist. As a result standard Mie theory, which assumes plane-wave illumination, is unable to predict adequately the details of the far-field intensity of the scattered light. ${ }^{7}$ In response to this inadequacy of Mie theory, Gouesbet and his collaborators devised a method to calculate theoretically the light scattered by a spherical particle that is illuminated by a polarized Gaussian laser beam $^{8,9}$ They found that the far-field intensity is determined by two sets of partial-wave amplitudes, the usual plane-wave Mie scattering amplitudes $a_{l}$ and $b_{l}$ and the partial-wave coefficients $A_{l m}$ and $B_{l m}$ of the incident beam.

In the formalism of Gouesbet et al., evaluation of the incident beam partial-wave coefficients $A_{l m}$ and $B_{l m}$ requires either three-dimensional integrations of the highly oscillatory radial component of the incident electric and magnetic fields over all space or the use of a finite-series technique. Barton et al.$^{10}$ simplified the computation of $A_{l m}$ and $B_{l m}$ by expressing the incident beam coefficients as two-dimensional surface integrals. They also applied the Gaussian beam formalism to the calculation of the interior fields of the spherical particle and to the near-field region and examined the production of morphological scattering resonances by on-axis and off-axis Gaussian beams. ${ }^{11.12}$

In the numerical evaluation of $A_{l m}$ and $B_{l m}$, it was noticed that the computed values of the coefficients exhibited a simple pattern that was reminiscent of the shape of the Gaussian beam. ${ }^{13-15}$ As a result it was believed that there existed some simple approximation to $A_{l m}$ and $B_{l m}$ in the $\lambda \rightarrow 0$ limit that could be used to simplify further the computation of the incident beam partial-wave coefficients. Such an approximation, accurate to a few parts in $10^{5}$, has been devised by Gouesbet et al., ${ }^{16}$ although we still lack a rigorous proof of it. This approximation is called the localization approximation since it resembles the localization principle for associating a geometrical light ray with a small group of partial waves in the plane-waveincidence scattering problem. ${ }^{17}$

If one uses numerical integration, the finite-series method, or the localization approximation for the evaluation of $A_{l m}$ and $B_{l m}$, the resulting far-field scattered intensity $I(\theta, \phi)$ for $2 \pi a / \lambda \gg 1$, where $a$ is the sphere radius, does not resemble the familiar $I(\theta)$ graphs for plane-wave incidence. ${ }^{18}$ The distinctive behavior of the scattered intensity for Gaussian beam incidence has been exploited by various authors. To observe the glare spots associated with high-order rainbows, Walker used a beam-blocking technique and on other occasions used an off-axis Gaussian laser beam (i.e., the center of the droplet does not lie on the symmetry axis of the beam) to illuminate his suspended liquid droplets. ${ }^{19-21}$ Similar off-axis beam experiments were performed earlier by Fahlan and Bryant to isolate the edge-ray contribution to glory scattering. ${ }^{22}$ From the viewpoint of ray theory, Walker's experiments used the offaxis Gaussian beam to amplify weak-scattering mechanisms relative to traditionally predominant ones in the following way. For plane-wave incidence with $2 \pi a / \lambda \gg 1$, van de Hulst ${ }^{23}$ and Bohren and Huffman ${ }^{24}$ have calculated that $>93 \%$ of the transverse-electric scattered intensity is produced by the geometrical light rays that are diffracted, specularly reflected, and transmitted by the sphere. More than $99.5 \%$ of the intensity is produced by these three ray processes and by the rays that emerge from the sphere after one or two internal reflections. Thus an exceedingly small fraction of the incident intensity is channeled into the production of high-order rainbows. But for off-axis Gaussian beam illumination, especially if the width of the beam is substantially less than the diameter of the particle, the rays that contribute to one or a number of the dominant processes are scattered to one side of the droplet, whereas the rays for some of the high-order rainbows are scattered to the other side. With the otherwise dominant contributions now absent, high-order rainbow visibility is increased.

In this paper I consider the idea that an off-axis Gaussian beam selectivity channels the scattered light of different physical processes to one side or the other of the spherical particle and use it to explain the novel features that are present in the Gaussian beam $I(\theta, \phi)$ graphs. The balance 
of this paper will proceed as follows. First, I briefly describe my notation, which is intermediate between that used by Gouesbet et al. ${ }^{8,9}$ and by Barton et al. ${ }^{10}$ Second, using this notation, I point out a number of the computational problems that must be addressed when one numerically calculates the far-field scattered intensity. Third, I perform a Debye-series decomposition of the Gaussian beam partial-wave scattering amplitudes $\alpha_{l m}$ and $\beta_{l m}$. The Debye series is the wave-theory analog of the geometrical-ray trajectories described above. ${ }^{25-29}$ This procedure decomposes the partial-wave scattering amplitudes into a series of terms that correspond to the spherical multipole partial waves that are diffracted, specularly reflected $(p=0)$, transmitted $(p=1)$, and transmitted after $p-1$ internal reflections within the sphere. I then compare the results of the Debye-series calculations with the complete Gaussian beam intensity graphs. Finally I describe an experiment whose purpose is to verify the dependence of rainbow intensity on the distance by which the laser beam is incident off axis.

\section{EXPRESSION FOR THE FAR-FIELD SCATTERED INTENSITY}

Consider a spherical particle of radius $a$ and refractive index $n$ whose center is at the origin of coordinates. Incident upon this particle is an electromagnetic wave of angular frequency $\omega$, wavelength $\lambda$, and propagation number

$$
k=\frac{2 \pi}{\lambda}=\frac{\omega}{c}
$$

traveling in the $z$ direction, polarized in the $x$ direction, and whose scalar radiation potential ${ }^{30}$ is of the form

$$
\Psi_{\text {inc }}(\mathbf{r}, t)=\psi_{\text {inc }}(\mathbf{r}) \exp (-i \omega t),
$$

where

$$
\nabla^{2} \psi_{\text {inc }}+k^{2} \psi_{\text {inc }}=0 .
$$

The transverse electric and transverse magnetic components of $\psi_{\text {inc }}$ are given by

$$
\begin{aligned}
\psi_{\text {inc }}^{\mathrm{TE}} & =\sum_{l m} i^{l} \frac{2 l+1}{2 l(l+1)} B_{l m} j_{l}(k r) P_{l}^{|m|}[\cos (\theta)] \exp (i m \varphi), \\
\psi_{\text {inc }}^{\mathrm{TM}} & =\sum_{l m} i^{l} \frac{2 l+1}{2 l(l+1)} A_{l m} j_{l}(k r) P_{l}^{|m|}[\cos (\theta)] \exp (i m \varphi),
\end{aligned}
$$

where $j_{l}$ and $n_{l}$ are spherical Bessel and spherical Neumann functions, $P_{l}^{|m|}$ are the associated Legendre polynomials, and $A_{l m}$ and $B_{l m}$ are the incident field partial-wave coefficients. By taking the appropriate derivatives of $\psi_{\text {inc }}$ one may calculate the $r, \theta$, and $\phi$ components of the incident electric and magnetic fields. ${ }^{30}$ For an arbitrary incident electromagnetic wave, the partial-wave coefficients $A_{l m}$ and $B_{l m}$ are given by ${ }^{10}$

$$
\begin{aligned}
A_{l m}= & \frac{(-i)^{l-1}}{2 \pi} \frac{k r}{j_{l}(k r)} \frac{(l-|m|) !}{(l+|m|) !} \int_{0}^{\pi} \sin (\theta) \mathrm{d} \theta \int_{0}^{2 \pi} \mathrm{d} \phi P_{l}^{|m|}[\cos (\theta)] \\
& \times \exp (-i m \varphi) E_{\mathrm{inc}}^{\mathrm{rad}}(r, \theta, \phi), \\
B_{l m}= & \frac{(-i)^{l-1}}{2 \pi} \frac{k r}{j_{l}(k r)} \frac{(l-|m|) !}{(l+|m|) !} \int_{0}^{\pi} \sin (\theta) \mathrm{d} \theta \int_{0}^{2 \pi} \mathrm{d} \phi P_{l}^{|m|}[\cos (\theta)] \\
& \times \exp (-i m \varphi) c B_{\text {inc }}^{\mathrm{rad}}(r, \theta, \phi),
\end{aligned}
$$

where the value of the radial coordinate $r$ is arbitrary, or by ${ }^{9,31}$

$$
\begin{aligned}
A_{l m}= & \frac{(i)^{l-1}}{\pi^{2}}(2 l+1) \frac{(l-|m|) !}{(l+|m|) !} \int_{0}^{\infty} k r \mathrm{~d}(k r) \int_{0}^{\pi} \sin (\theta) \mathrm{d} \theta \\
& \times \int_{0}^{2 \pi} \mathrm{d} \phi j_{l}(k r) P_{l}^{|m|}[\cos (\theta)] \exp (-i m \phi) E_{\mathrm{inc}}^{\mathrm{rad}}(r, \theta, \phi), \\
B_{l m}= & \frac{(i)^{l-1}}{\pi^{2}}(2 l+1) \frac{(l-|m|) !}{(l+|m|) !} \int_{0}^{\infty} k r \mathrm{~d}(k r) \int_{0}^{\pi} \sin (\theta) \mathrm{d} \theta \\
& \times \int_{0}^{2 \pi} \mathrm{d} \phi j_{l}(k r) P_{l}^{|m|}[\cos (\theta)] \exp (-i m \phi) c B_{\mathrm{inc}}^{\mathrm{rad}}(r, \theta, \phi) .
\end{aligned}
$$

Before we continue, Eqs. (5) and (6) warrant a number of comments. First, in Eqs. (4)-(6) I used the complete set of angular functions $P_{l}^{|m|}[\cos (\theta)] \exp (\operatorname{im} \varphi)$, as did Gouesbet et al., ${ }^{8,9}$ instead of the spherical harmonics $Y_{l}^{m}(\theta, \phi)$ used by Barton et al. ${ }^{10}$ I prefer the angular functions of Gouesbet et al. because of their computational convenience. In particular, since the associated Legendre function differential equation depends only on $m^{2}$ rather than on $m,{ }^{32} \mathrm{I}$ can employ the same function $P_{l}^{|m|}$ for both positive and negative $m$ rather than renormalizing the associated Legendre polynomials for negative $m$. Second, since the scalar radiation potential $\psi_{\mathrm{inc}}$ is assumed to be a solution of Eq. (3), the expansion coefficients $A_{l m}$ and $B_{l m}$ are unique, i.e., since $E_{\text {inc }}^{\text {rad }}$ and $B_{\text {inc }}^{\text {rad }}$ are derived from $\psi_{\text {inc }}$, the values of $A_{l m}$ and $B_{l m}$ in Eqs. (5) are independent of the radial evaluation point $r$ that is chosen. This means that the two-dimensional integrals of Eqs. (5) must be proportional to $j_{l}(k r) / k r$ so as to cancel the $k r / j_{l}(k r)$ factor that multiplies them. From a numerical methods viewpoint, the constancy of $A_{l m}$ and $B_{l m}$ when $r$ is varied provides a test of the stability of one's numerical integration routines. Once I determined the grid sizes for the $\theta$ and $\phi$ integrations so that the computed values of $A_{l m}$ and $B_{l m}$ were independent of $r$, I chose the value $r=a$ for all subsequent computations. The value $r=a$ was also employed by Barton et al. ${ }^{10}$ Finally, in their derivation of the threedimensional integrals for $A_{l m}$ and $B_{l m}$, Gouesbet et al. ${ }^{33}$ used the orthogonality relation

$$
\int_{0}^{\infty} \mathrm{d}(k r) j_{l}(k r) j_{l^{\prime}}(k r)=\frac{\pi}{2(2 l+1)} \delta_{l l} .
$$

This equation is not correct since $j_{l}(k r)$ and $j_{l^{\prime}}(k r)$ are not orthogonal for $l \neq l^{\prime}$ and $l+l^{\prime}=$ even. Despite the incorrectness of Eq. (7), their final result for $A_{l m}$ and $B_{l m}$ and my Eqs. (6) are correct.

Once the incident beam is expanded into partial waves, the derivation of the partial-wave scattering amplitudes is analogous to the plane-wave incidence derivation. If we take

$$
\begin{aligned}
\psi_{\text {scattered }}^{\mathrm{TE}}= & \sum_{l=0}^{\infty} \sum_{m=-l}^{l} i^{l} \frac{2 l+1}{l(l+1)}\left(-\beta_{l m}\right) h_{l}{ }^{(1)}(k r) P_{l}^{|m|}[\cos (\theta)] \\
& \times \exp (i m \varphi), \\
\psi_{\text {scattered }}^{\mathrm{TM}}= & \sum_{l=0}^{\infty} \sum_{m=-l}^{l} i^{l} \frac{2 l+1}{l(l+1)}\left(-\alpha_{l m}\right) h_{l}^{(1)}(k r) P_{l}^{|m|}[\cos (\theta)] \\
& \times \exp (i m \varphi)
\end{aligned}
$$

for the partial-wave decomposition of the scattered wave scalar radiation potential and

$$
\begin{aligned}
\psi_{\text {inside }}^{\mathrm{TE}}= & \sum_{l=0}^{\infty} \sum_{m=-l}^{l} i^{l} \frac{2 l+1}{l(l \neq 1)}\left(n \delta_{l m}\right) j_{l}(n k r) P_{l}^{|m|}[\cos (\theta)] \\
& \times \exp (i m \varphi) \\
\psi_{\text {inside }}^{\mathrm{TM}}= & \sum_{l=0}^{\infty} \sum_{m=-l}^{l} i^{l} \frac{2 l+1}{l(l+1)}\left(n \gamma_{l m}\right) j_{l}(n k r) P_{l}^{|m|}[\cos (\theta)] \\
& \times \exp (i m \varphi)
\end{aligned}
$$


for the partial-wave decomposition of the interior scalar radiation potential, the continuity of $E_{\theta}, E_{\phi}, B_{\theta}, B_{\phi}$ at $r=a$ gives

$$
\begin{aligned}
& \lim _{r \rightarrow \infty} \mathbf{E}_{\text {scattered }}(r, \theta, \phi)=\frac{i \exp (i k r)}{k r}\left[S_{2}(\theta, \phi) \hat{u}_{\theta}-S_{1}(\theta, \phi) \hat{u}_{\phi}\right], \\
& \lim _{r \rightarrow \infty} \mathbf{B}_{\text {scattered }}(r, \theta, \phi)=\frac{i \exp (i k r)}{c k r}\left[S_{1}(\theta, \phi) \hat{u}_{\theta}+S_{2}(\theta, \phi) \hat{u}_{\phi}\right],
\end{aligned}
$$

$$
\lim _{r \rightarrow \infty} I_{\text {scattered }}(r, \theta, \phi)=\frac{1}{2 \mu_{0} c} \frac{1}{k^{2} r^{2}}\left[\left|S_{1}(\theta, \phi)\right|^{2}+\left|S_{2}(\theta, \phi)\right|^{2}\right],
$$

where

$$
\begin{aligned}
& S_{1}(\theta, \phi)= \sum_{l=0}^{\infty} \sum_{m=-l}^{l} \frac{2 l+1}{2 l(l+1)}\left[-i m \alpha_{l m} \pi_{l}^{|m|}(\theta)+\beta_{l m} \tau_{l}^{|m|}(\theta)\right] \\
& \times \exp (i m \varphi) \\
& S_{2}(\theta, \phi)= \sum_{l=0}^{\infty} \sum_{m=-l}^{l} \frac{2 l+1}{2 l(l+1)}\left[i m \beta_{l m} \pi_{l}^{|m|}(\theta)+\alpha_{l m} \tau_{l}^{|m|}(\theta)\right] \\
& \times \exp (i m \varphi) \\
& \pi_{l}^{|m|}(\theta)=\frac{1}{\sin \theta} P_{l}^{|m|}[\cos (\theta)] \\
& \tau_{l}^{|m|}(\theta)=\frac{\mathrm{d}}{\mathrm{d} \theta} P_{l}^{|m|}[\cos (\theta)] \\
& \alpha_{l m}=A_{l m} a_{l} \\
& \beta_{l m}=B_{l m} b_{l}
\end{aligned}
$$

and where $a_{l}$ and $b_{l}$ are the plane-wave partial wave scattering amplitudes of Mie theory. Equations (10)-(14), with either Eqs. (5) or (6) used to evaluate the incident beam partial-wave coefficients, represent the formal solution to the scattering of arbitrary incident beam by a spherical particle. For plane-wave incidence with the initial electric field polarized in the $x$ direction, we obtain

$$
\begin{aligned}
E_{\mathrm{inc}}^{\mathrm{rad}}(r, \theta, \phi) & =\exp [i k r \cos (\theta)] \sin (\theta) \cos (\phi), \\
c B_{\mathrm{inc}}^{\mathrm{rad}}(r, \theta, \phi) & =\exp [i k r \cos (\theta)] \sin (\theta) \sin (\phi)
\end{aligned}
$$

As a result Eqs. (5) give

$$
\begin{aligned}
& A_{l m}= \begin{cases}1 & \text { if } m= \pm 1 \\
0 & \text { if otherwise }\end{cases} \\
& B_{l m}=\left\{\begin{array}{ll}
\mp i & \text { if } m= \pm 1 \\
0 & \text { if otherwise }
\end{array},\right.
\end{aligned}
$$

and Eqs. (12) reduce to the familiar expressions

$$
\begin{aligned}
& S_{1}(\theta, \phi)=\sum_{l=1}^{\infty} \frac{2 l+1}{l(l+1)}\left[a_{l} \pi_{l}{ }^{1}(\theta)+b_{l} \tau_{l}{ }^{1}(\theta)\right] \sin (\phi), \\
& S_{2}(\theta, \phi)=\sum_{l=1}^{\infty} \frac{2 l+1}{l(l+1)}\left[a_{l} \tau_{l}{ }^{1}(\theta)+b_{l} \pi_{l}{ }^{1}(\theta)\right] \cos (\phi) .
\end{aligned}
$$

If the arbitrary incident beam is a focused Gaussian beam, the Davis formalism may be used to express $E_{\mathrm{inc}}^{\mathrm{rad}}$ and $B_{\text {inc }}^{\text {rad }}$ as a series expansion in powers of $s=\lambda / 2 \pi w$, where $w$ is the half-width of the incident beam. ${ }^{34}$ For many applications $s \ll 1$, and the Davis first-order formalism is adequate. If the beam is traveling in the $z$ direction and the focal waist is at the origin, the Davis first-order formalism gives the $\hat{u}_{x}$ polarized incident fields as

$$
\begin{aligned}
\mathbf{E}_{\mathrm{inc}}= & \frac{\exp (i k z)}{1+(2 i z s) / w} \exp \left[\frac{-\left(x^{2}+y^{2}\right) / w^{2}}{1+(2 i z s) / w}\right] \\
& \times\left[\hat{u}_{x}-\frac{2 i x s}{w}\left(1+\frac{2 i z s}{w}\right)^{-1} \hat{u}_{z}\right], \\
E_{\mathrm{inc}}^{\mathrm{rad}}= & E_{\mathrm{inc}}^{x} \sin (\theta) \cos (\phi)+E_{\mathrm{inc}}^{z} \cos (\theta), \\
c \mathbf{B}_{\mathrm{inc}}= & \frac{\exp (i k z)}{1+(2 i z s) / w} \exp \left[\frac{-\left(x^{2}+y^{2}\right) / w^{2}}{1+(2 i z s) / w}\right] \\
& \times\left[\hat{u}_{y}-\frac{2 i y s}{w}\left(1+\frac{2 i z s}{w}\right)^{-1} \hat{u}_{z}\right], \\
c B_{\mathrm{inc}}^{\mathrm{rad}}= & c B_{\mathrm{inc}}^{y} \sin (\theta) \sin (\phi)+c B_{\mathrm{inc}}^{z} \cos (\theta) .
\end{aligned}
$$

The various terms of Eqs. (19) and (21) have the following physical interpretations. The dying exponential part of

$$
\exp \left[\frac{-\left(x^{2}+y^{2}\right) / w^{2}}{1+(2 i z s) / w}\right]
$$

represents the Gaussian falloff of half-width $w$ of the fields in the $z=0$ focal plane. The oscillatory portion of the exponential represents an approximation to the curvature of the planes of constant phase to either side of the focal plane. The magnitude of the denominator of the argument of the exponential describes the widening of the Gaussian beam before and after the focal plane. The overall denominators of $E_{\text {inc }}^{x}$ and $B_{\text {inc }}^{y}$ describe the decrease in amplitude that accompanies the widening of the beam away from the focal plane. Finally, $E_{\text {inc }}^{z}$ and $B_{\text {inc }}^{z}$ are a result of the curvature of the planes of constant phase to either side of the focal plane along with requirement that the directions of the fields be transverse. This behavior is pictorially represented in Fig. 1 . If the beam focal point is located at $\left(x_{0}, y_{0}, z_{0}\right)$ rather than at the origin, one makes the replacements

$$
\begin{aligned}
& x \rightarrow x-x_{0}, \\
& y \rightarrow y-y_{0}, \\
& z \rightarrow z-z_{0}
\end{aligned}
$$

in Eqs. (19) and (21). For a tightly focused laser beam where $s$ is not necessarily small, more terms in the powerseries expansions of $\mathbf{E}_{\text {inc }}$ and $\mathbf{B}_{\text {inc }}$ are required, producing the so-called Davis third-order ${ }^{34}$ or Davis fifth-order ${ }^{35}$ formulas, depending on the number of terms that are retained.

If the center of the spherical particle is on the symmetry axis of a first-order Davis beam (i.e., on-axis incidence), the Gaussian beam scattering formulas of Eqs. (5) and (10)-(14) simplify almost to the extent that they do for plane-wave incidence. Using Eqs. (19)-(22), we can determine that the radial components of the first-order Davis incident fields on axis are of the form

$$
\begin{aligned}
E_{\text {inc }}^{\mathrm{rad}} & =\exp [i k r \cos (\theta)] f(k r, \theta) \sin (\theta) \cos (\phi), \\
c B_{\text {inc }}^{\mathrm{rad}} & =\exp [i k r \cos (\theta)] f(k r, \theta) \sin (\theta) \sin (\phi) .
\end{aligned}
$$




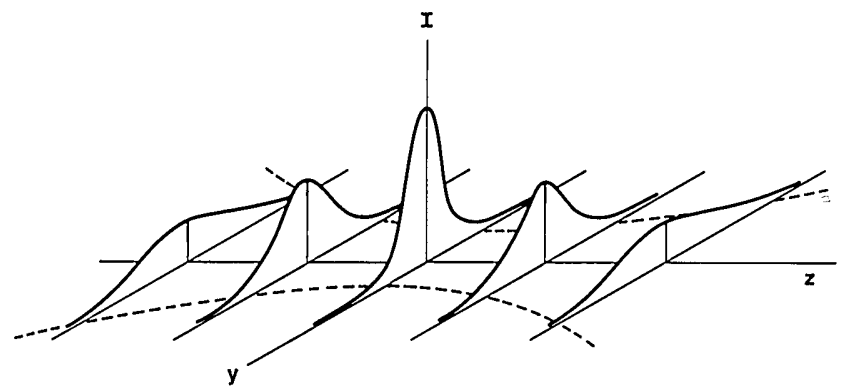

(a)

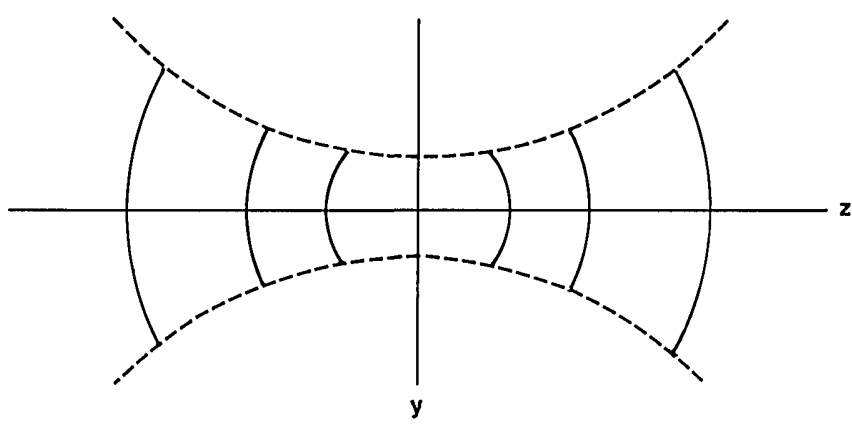

(b)

Fig. 1. (a) Intensity of a Gaussian beam as a function of position in the $y z$ plane. The beam is propagating in the positive $z$ direction, and the center of the focal waist is at $y=z=0$. The dashed curves represent the nominal width of the beam as a function of $z$. (b) The planes of constant phase of the Gaussian beam as a function of position in the $y z$ plane. The curvature of the planes of constant phase for $z<0$ and $z>0$ is due to the convergence and divergence of the beam on each side of the focal plane. The dashed curves represent the nominal width of the beam as a function of $z$.

Substituting Eqs. (24) into Eqs. (5) and Eqs. (5) into Eqs. (10)-(14), only $m= \pm 1$ contribute, and

$$
\begin{aligned}
& S_{1}(\theta, \phi)=\sum_{l=1}^{\infty} \frac{2 l+1}{l(l+1)} I_{l}\left[a_{l} \pi_{l}{ }^{1}(\theta)+b_{l} \tau_{l}{ }^{1}(\theta)\right] \sin (\phi), \\
& S_{2}(\theta, \phi)=\sum_{l=1}^{\infty} \frac{2 l+1}{l(l+1)} I_{l}\left[a_{l} \pi_{l}{ }^{1}(\theta)+b_{l} \pi_{l}{ }^{1}(\theta)\right] \cos (\phi),
\end{aligned}
$$

where

$$
\begin{aligned}
I_{l}= & \frac{(-i)^{l-1}}{2} \frac{k r}{j_{l}(k r)} \frac{1}{l(l+1)} \\
& \times \int_{0}^{\pi} \sin ^{2}(\theta) \mathrm{d} \theta f(k r, \theta) \exp [i k r \cos (\theta)] P_{l}^{1}[\cos (\theta)] .
\end{aligned}
$$

This simplification does not occur if the Gaussian beam is incident off axis, and the simplification assumes a somewhat more elaborate form for the fifth-order Davis beam on axis. ${ }^{36}$

\section{NUMERICAL CONSIDERATIONS FOR CALCULATING THE FAR-FIELD SCATTERED INTENSITY}

The computational details that must be addressed in the implementation of Eqs. (5) and (10)-(14) fall into four general classes: (a) the computation of the plane-wave incidence partial-wave scattering amplitudes $a_{l}$ and $b_{l}$, (b) the computation of the incident beam partial-wave coefficients $A_{l m}$ and $B_{l m}$, (c) the computation of the angular functions $\pi_{l}^{|m|}(\theta)$ and $\tau_{l}^{\mid(\mid)}(\theta)$, and (d) the truncation of the double sum in $l$ and $m$. We need not address the computation of $a_{l}$ and $b_{l}$ since the issues involved in their calculation have been extensively analyzed in the literature. ${ }^{37-39}$

In evaluating $A_{l m}$ and $B_{l m}$, we must first compute the $P_{l}^{|m|} \cos (\theta)$. We do this for $m \geq 1$ by starting with

$$
\begin{aligned}
P_{l-1}^{l}[\cos (\theta)] & =0, \\
P_{l}^{l}[\cos (\theta)] & =1 \times 3 \times 5 \ldots(2 l-1) \sin ^{l}(\theta) \\
& \equiv(2 l-1) ! ! \sin ^{l}(\theta)
\end{aligned}
$$

and using the upward-recursion relation

$$
\begin{aligned}
P_{l+1}{ }^{m}[\cos (\theta)]= & \frac{2 l+1}{l+1-m} \cos (\theta) P_{l}^{m}[\cos (\theta)] \\
& -\frac{l+m}{l+1-m} P_{l-1}{ }^{m}[\cos (\theta)] .
\end{aligned}
$$

For $m=0$, we start with

$$
\begin{aligned}
& P_{0}[\cos (\theta)]=1, \\
& P_{1}[\cos (\theta)]=\cos (\theta)
\end{aligned}
$$

and use the upward-recursion relation

$$
P_{l+1}[\cos (\theta)]=\frac{2 l+1}{l+1} \cos (\theta) P_{l}[\cos (\theta)]-\frac{l}{l+1} P_{l-1}[\cos (\theta)] .
$$

Another consideration in evaluating the two-dimensional integrals of Eqs. (5) is the choice of a grid size for the $\theta$ and $\phi$ integrations. When Eqs. (5) are evaluated at $r=a$, the most rapidly varying term in the Davis first-order fields is the exp[ika $\cos (\theta)]$ factor of Eqs. (19) and (21). This factor undergoes $k a / \pi$ cycles of oscillation in the interval $0 \leq$ $\theta \leq \pi$, with the oscillations being slower near $\theta=0, \pi$ and faster near $\theta=\pi / 2$. Thus an angular grid size of

$$
\Delta \theta=\pi /(10 k a)
$$

gives 31 samplings per cycle of the $\exp [i k a \cos (\theta)]$ factor averaged over the entire interval $0 \leq \theta \leq \pi$ and 20 samplings per cycle for the most rapidly oscillating region at $\theta \approx \pi / 2$. In addition, the sum over partial waves $l$ is truncated in traditional Mie programs at ${ }^{39}$

$$
l_{\max }=k a+4.05(k a)^{1 / 3}+2,
$$

which for large spheres is approximately $l_{\max } \approx k a$. The associated Legendre polynomial in the integrand of Eqs. (5) with the most rapid oscillations is then $P_{l \max }^{|m|}[\cos (\theta)]$. This function has $\sim l_{\max } / 2$ oscillations in the interval $0 \leq \theta \leq \pi$, and the angular grid size of Eq. (31) again gives 20 samplings per oscillation of $P_{l \max }|m|$.

The most rapid $\phi$ dependence in the integrand of Eqs. (5) is the $\exp (-i m \phi)$ factor, which has $m$ cycles of oscillation in the interval $0 \leq \phi \leq 2 \pi$. An angular grid size of

$$
\Delta \phi=\frac{2 \pi}{100+20 m}
$$

samples the $\phi$ dependence at a much finer scale than does Eq. (31) for the $\theta$ dependence. For plane-wave incidence, 
the use of Eqs. (31) and (33) for the angular grid in Eqs. (5) produced numerical values of the coefficients that differed from the analytical results of Eqs. (16) and (17) by $<3$ parts in $10^{6}$.

For the evaluation point $r=a$, the spherical Bessel functions appearing in Eqs. (5) may be calculated by upward recursion in double precision by using

$$
\begin{aligned}
j_{0}(k a) & =\frac{\sin (k a)}{k a} \\
j_{1}(k a) & =\frac{\sin (k a)}{(k a)^{2}}-\frac{\cos (k a)}{k a}, \\
j_{l+1}(k a) & =\frac{2 l+1}{k a} j_{l}(k a)-j_{l-1}(k a)
\end{aligned}
$$

Varying the evaluation parameter $r$ to values greater than $a$ preserves the accuracy of their upward-recursion evaluation by using Eqs. (34) and (35). It also provides the above-mentioned test of the stability of the numerical integration routine and determines the value of $s$ for tightly focused laser beams at which the first-order Davis formalism no longer represents an adequate approximation to a solution of Eq. (3). Numerical tests on Eq. (26) showed that the Davis first-order beam parameterization of Eqs. (19)-(22) is valid to 5 parts in $10^{5}$ in the evaluation of $I_{l}$ for $s=0.0067$ (i.e., $w=12 \mu \mathrm{m}$ at $\lambda=0.5145 \mu \mathrm{m}$ ). The accuracy decreases to 30 parts in $10^{5}$ for $s=0.01$. For narrow beams it becomes necessary to use the Davis fifth-order formalism to obtain few-parts-in- $10^{5}$ accuracy in the evaluation of $A_{l m}, B_{l m}$, and $I_{l}$. Another test of the accuracy of the integration routine for the evaluation of $A_{l m}$ and $B_{l m}$ is provided by the symmetry properties that these coefficients have for a Davis first-order beam. It can be analytically shown by examining the $\phi$ integration that, for $x_{0} \neq 0, y_{0}=0$, and $m \geq 1$,

$$
\begin{aligned}
& A_{l-m}=A_{l m}, \\
& B_{l-m}=-B_{l m}
\end{aligned}
$$

and, for $x_{0}=0, y_{0} \neq 0$, and $m \geq 1$,

$$
\begin{aligned}
& A_{l-m}=\left\{\begin{array}{rl}
-A_{l m} & \text { for } m=\text { even } \\
A_{l m} & \text { for } m=\text { odd }
\end{array},\right. \\
& B_{l-m}=\left\{\begin{array}{rl}
B_{l m} & \text { for } m=\text { even } \\
-B_{l m} & \text { for } m=\text { odd }
\end{array} .\right.
\end{aligned}
$$

The angular functions $\pi_{l}^{|m|}(\theta)$ and $\tau_{l}^{|m|}(\theta)$ are computed in the following way. For $m \geq 1$ we start with

$$
\begin{aligned}
\pi_{l-1}^{l}(\theta) & =0 \\
\pi_{l}^{l}(\theta) & =(2 l-1) ! ! \sin ^{l-1}(\theta)
\end{aligned}
$$

and use the upward-recursion relation

$$
\begin{aligned}
\pi_{l+1}^{|m|}(\theta)= & \frac{2 l+1}{l+1-|m|} \cos (\theta) \pi_{l}^{|m|}(\theta) \\
& -\frac{l+|m|}{l+1-|m|} \pi_{l-1}^{|m|}(\theta)
\end{aligned}
$$

The function $\tau_{l}^{|m|}(\theta)$ is then computed by the upwardrecursion relation

$$
\tau_{l}^{|m|}(\theta)=l \cos (\theta) \pi_{l}^{|m|}(\theta)-(l+|m|) \pi_{l-1}^{|m|}(\theta) .
$$

For $m=0, \pi_{l}{ }^{0}(\theta)$ diverges at $\theta=0, \pi$. However, this function does not contribute to $S_{1}$ and $S_{2}$ since only the quantity $i m \pi_{l}^{|m|}(\theta)$ appears in Eqs. (12).

To compute $\tau_{l}{ }^{0}(\theta)$, we calculate $P_{l} \cos (\theta)$ by means of Eqs. (29) and (30) and $P_{l}^{\prime}[\cos (\theta)]$ by

$$
\begin{aligned}
P_{0}^{\prime}[\cos (\theta)] & =0, \\
P_{l}^{\prime}[\cos (\theta)] & =l P_{l-1}[\cos (\theta)]+\cos (\theta) P_{l-1}^{\prime}[\cos (\theta)]
\end{aligned}
$$

Then $\tau_{l}^{0}$ is given by

$$
\tau_{l}^{0}(\theta)=-\sin (\theta) P_{l}^{\prime}[\cos (\theta)]
$$

The angular functions have the following properties: When

$P_{l}^{|m|}[\cos (\theta)] \rightarrow \frac{(l+|m|) !}{(l-|m|) !}\left(l+\frac{1}{2}\right)^{-|m|} J_{|m|}\left[\left(l+\frac{1}{2}\right) \theta\right]$

is used for $\theta \rightarrow 0, \pi$ the angular functions approach

$$
\tau_{l}^{|m|}(\theta) \approx|m| \pi_{l}^{|m|}(\theta) \rightarrow \frac{1}{2} \frac{(l+|m|) !}{(l-|m|) !} \frac{(\theta / 2)^{|m|-1}}{(|m|-1) !}
$$

in this limit. This ensures that both $\alpha_{l m}$ and $\beta_{l m}$ contribute equally to diffraction, as was the case for plane-wave incidence. For $l \gg 1$ and away from $\theta=0, \pi$ with the use of the asymptotic form

$$
\begin{aligned}
P_{l}^{|m|}[\cos (\theta)] \rightarrow & \left(\frac{2}{\pi}\right)^{1 / 2}[\sin (\theta)]^{-1 / 2} l^{|m|-1 / 2} \\
& \times \cos \left[\left(l+\frac{1}{2}\right) \theta+\frac{|m| \pi}{2}-\frac{\pi}{4}\right],
\end{aligned}
$$

the angular functions approach

$$
\begin{aligned}
\pi_{l}^{|m|}(\theta) \rightarrow & \left(\frac{2}{\pi}\right)^{1 / 2}[\sin (\theta)]^{-3 / 2} l^{|m|-1 / 2} \\
& \times \cos \left[\left(l+\frac{1}{2}\right) \theta+\frac{|m| \pi}{2}-\frac{\pi}{4}\right], \\
\pi_{l}^{|m|}(\theta) \rightarrow & \left(\frac{2}{\pi}\right)^{1 / 2} \sin (\theta)^{-1 / 2} l^{|m|+1 / 2} \\
& \times \sin \left[\left(l+\frac{1}{2}\right) \theta+\frac{|m| \pi}{2}-\frac{\pi}{4}\right] \approx l \pi_{l}^{|m|}(\theta) .
\end{aligned}
$$

Then, just as for plane-wave incidence $\tau_{l}^{|m|} \gg \pi_{l}^{|m|}$ and the TE (TM) partial-wave scattering amplitude, $b_{l}\left(a_{l}\right)$ in 
$S_{1}\left(S_{2}\right)$ becomes associated with the TE (TM) polarized light rays.

In evaluating the double sum over $l$ and $m$ in Eqs. (12), it is of great value to interchange the order of the sums and obtain

$$
\begin{aligned}
S_{1}(\theta, \phi)= & \sum_{l=1}^{l \max } \frac{2 l+1}{2 l(l+1)} B_{l 0} b_{l} \tau_{l}^{0}(\theta) \\
& +\sum_{m=1}^{\infty} \sum_{l=m}^{l \max } \frac{2 l+1}{2 l(l+1)} i m a_{l} \pi_{l}^{m}(\theta) \\
& \times\left[-A_{l m}^{+} \exp (i m \phi)+A_{l m}^{-} \exp (-i m \phi)\right] \\
& +\sum_{m=1}^{\infty} \sum_{l=m}^{\max } \frac{2 l+1}{2 l(l+1)} b_{l} \tau_{l}^{m}(\theta) \\
& \times\left[B_{l m}^{+} \exp (i m \phi)+B_{l m}^{-} \exp (-i m \phi]\right) \\
S_{2}(\theta, \phi)= & \sum_{l=1}^{l \max } \frac{2 l+1}{2 l(l+1)} A_{l 0} a_{l} \tau_{l}^{0}(\theta) \\
& +\sum_{m=1}^{\infty} \sum_{l=m}^{l \max } \frac{2 l+1}{2 l(l+1)} a_{l} \tau_{l}^{m}(\theta) \\
& \times\left[A_{l m}^{+} \exp (i m \phi)+A_{l m}^{-} \exp (-i m \phi)\right] \\
& +\sum_{m=1}^{\infty} \sum_{l=m}^{l \max } \frac{2 l+1}{2 l(l+1)} i m b_{l} \pi_{l}^{m}(\theta) \\
& \times\left[B_{l m}^{+} \exp (i m \phi)-B_{l m}^{-} \exp (-i m \phi)\right]
\end{aligned}
$$

where

$$
\begin{array}{ll}
A_{l m}^{+} \equiv A_{l m} & \text { for } m \geq 1, \\
A_{l m}^{-} \equiv A_{l-m} & \text { for } m \geq 1
\end{array}
$$

integrals of Eqs. (26), (5), and (6), respectively. Since the partial-wave scattering amplitudes $\alpha_{l m}$ and $\beta_{l m}$ obey Eqs. (14), the localization approximation for $A_{l m}$ and $B_{l m}$ may be used for morphological scattering resonances, near fields, and interior fields as well as for far-field scattering. For an on-axis laser beam, the approximation in our notation takes the form

$$
I_{l} \approx \frac{\exp \left[\frac{-(l+1 / 2)^{2} / k^{2} w^{2}}{1-\left(2 i z_{0} s\right) / w}\right]}{1-\left(2 i z_{0} s\right) / w} .
$$

A derivation of this expression is given in Appendix A. Relation (50) may be physically interpreted as the Gaussian amplitude profile of the incident rays that strike the surface of the sphere at the various angles of incidence. ${ }^{40}$ For an off-axis beam, the localization approximation is $^{16}$

$$
\begin{aligned}
A_{l m} \approx & \frac{K_{l m}}{2} \frac{\exp \left\{\frac{-\left[k^{2} x_{0}{ }^{2}+k^{2} y_{0}{ }^{2}+(l+1 / 2)^{2}\right] / k^{2} w^{2}}{1-\left(2 i z_{0} s\right) / w}\right\}}{1-\left(2 i z_{0} s\right) / w} \\
& \times \sum_{j=0}^{\infty} \sum_{p=0}^{j}\left(\Psi_{j p} \delta_{j-2 p+1, m}+\Psi_{j p} \delta_{j-2 p-1, m}\right) \exp \left(-i k z_{0}\right), \\
B_{l m} \approx & \frac{K_{l m}}{2 i} \frac{\exp \left\{\frac{-\left[k^{2} x_{0}{ }^{2}+k^{2}{y_{0}}^{2}+(l+1 / 2)^{2}\right] / k^{2} w^{2}}{1-\left(2 i z_{0} s\right) / w}\right\}}{1-\left(2 i z_{0} s\right) / w} \\
& \times \sum_{j=0}^{\infty} \sum_{p=0}^{j}\left(\Psi_{j p} \delta_{j-2 p+1, m}-\Psi_{j p} \delta_{j-2 p-1, m}\right) \exp \left(-i k z_{0}\right),
\end{aligned}
$$

and similarly for $B_{l m}^{ \pm}$. Interchanging the order of the

where

$$
K_{l m}= \begin{cases}\frac{2 l(l+1)}{2 l+1}(2 i) & \text { for } m=0 \\ \left(\frac{2}{2 l+1}\right)^{|m|-1}(2 i)(-1)^{|m| / 2} & \text { for } m=\text { even and }|m| \geq 2, \\ \left(\frac{2}{2 l+1}\right)^{|m|-1}(2)(-1)^{|m| / 2-1 / 2} & \text { for } m=\text { odd }\end{cases}
$$

$$
\Psi_{j p}=\frac{\left(x_{0}-i y_{0}\right)^{j-p}\left(x_{0}+i y_{0}\right)^{p}}{(j-p) ! p !}\left[\frac{(l+1 / 2) s / w}{1-\left(2 i z_{0} s\right) / w}\right]^{j} .
$$

sums is useful because the scattering amplitudes $S_{1}(\theta, \phi)$ and $S_{2}(\theta, \phi)$ are often rapidly convergent in $m$. In relations (44) and (46) the angular functions $\pi_{l}^{|m|}$ and $\tau_{l}^{|m|}$ grow with increasing $m$. However, it can be shown that $A_{l m}$ and $B_{l m}$ decrease with increasing $m$ as

$$
A_{l m} \sim B_{l m} \sim \exp \left[-(l+1 / 2)^{2} / k^{2} w^{2}\right]\left(\frac{a}{w}\right)^{|m|-1} \frac{s^{|m|-1}}{(|m|-1) !} .
$$

If $A_{l m}$ and $B_{l m}$ decrease faster than $\pi_{l}^{|m|}$ and $\tau_{l}^{|m|}$ increase, the sums over $m$ in Eqs. (47) will be rapidly convergent. For example, for $\lambda=0.5145 \mu \mathrm{m}, w=20 \mu \mathrm{m}, a=$ $43.3 \mu \mathrm{m}, n=1.33, x_{0}=z_{0}=0$, and $y_{0}=40 \mu \mathrm{m}$ (i.e., far off-axis incidence), we have $A_{l 0} \sim B_{l 0} \sim 10^{2}, A_{l 1} \sim B_{l 1} \sim$ $10^{-2}, A_{l 2} \sim B_{l 2} \sim 10^{-4}, A_{l 3} \sim B_{l 3} \sim 10^{-6}, A_{l 4} \sim B_{l 4} \sim 10^{-9}$, and $A_{l 5} \sim B_{l 5} \sim 10^{-12}$. Thus truncation of the $m$ sum at $m_{\max }=5$ introduces little error. This value of $m_{\max }$ was used in all the calculations reported in Section 4.

Finally, Gouesbet's localization approximation ${ }^{16}$ permits one to evaluate $A_{l m}$ and $B_{l m}$ to few-parts-in- $10^{5}$ accuracy without performing the one-, two-, or three-dimensional
The stationary-phase method of deriving relation (50) that is presented in Appendix A is not generalizable to the case of off-axis incidence. This is discussed in Appendix A. Furthermore, the form of the localization approximation for the Davis third-order and Davis fifth-order beams is not known at present. Convergence of the localization approximation to $A_{l m}$ and $B_{l m}$ to 10 parts in $10^{5}$ (and often to 1 part in $10^{5}$ ) of their values obtained by numerical integration of Eqs. (5) was achieved by truncating the $j$ sum at $j=21$. For all the results reported in Section 4, the values of $A_{l m}$ and $B_{l m}$ were calculated by means of the localization approximation.

\section{FAR-FIELD SCATTERED INTENSITY $I(\theta, \phi)$ FOR GAUSSIAN BEAM INCIDENCE}

The results that have already appeared for $I(\theta, \phi)$ for the scattering of a Gaussian laser beam by a spherical particle 


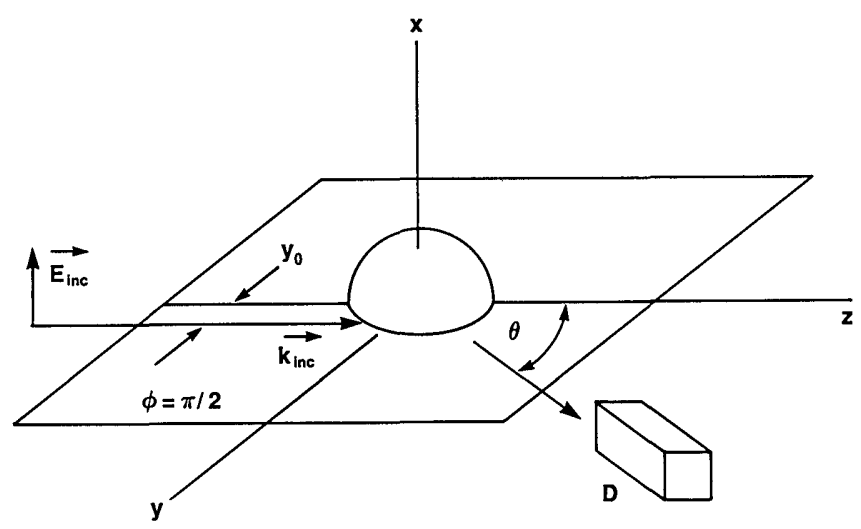

Fig. 2. Scattering geometry. A Gaussian laser beam polarized in the $x$ direction propagating parallel to the $z$ axis and displaced from it by the distance $y_{0}$ in the $y z$ plane is incident upon a spherical particle. The scattered light is measured at the scattering angle $\theta$ to the right of the $z$ direction in the $y z$ plane $(\phi=\pi / 2)$ by the detector $D$.

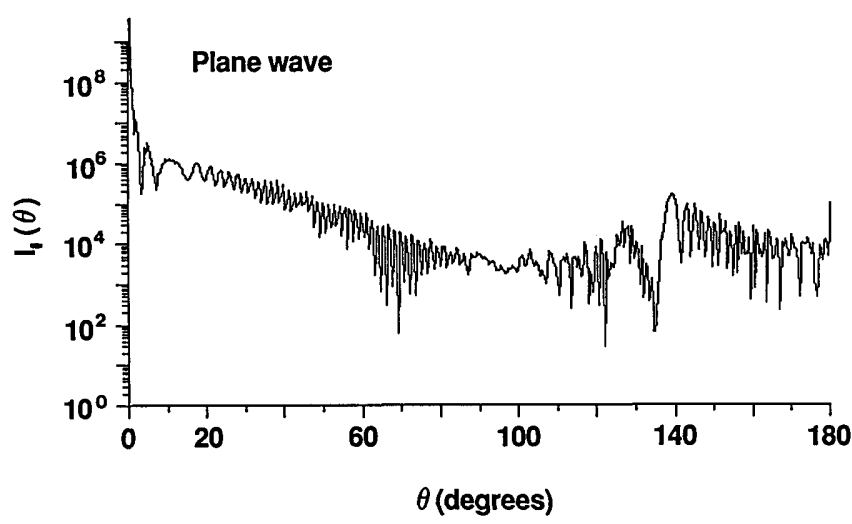

Fig. 3. Far-field scattered intensity $I_{1}(\theta)=\left|S_{1}(\theta)\right|^{2}$ as a function of scattering angle for a plane wave with $\lambda=0.5145 \mu \mathrm{m}$ and polarized in the $x$ direction incident upon a spherical particle with $a=43.3 \mu \mathrm{m}$ and $n=1.33$.

look quite different from the $I(\theta)$ graphs for plane-wave incidence. ${ }^{18}$ In this section I interpret the various features of the $I(\theta, \phi)$ curves for both an on-axis and an off-axis $\hat{u}_{x}$ polarized Davis first-order Gaussian laser beam of $w=$ $20 \mu \mathrm{m}$ and $\lambda=0.5145 \mu \mathrm{m}$ that is incident upon a spherical particle of $a=43.3 \mu \mathrm{m}$ and $n=1.33$. The geometry is shown in Fig. 2. The scattered intensity is measured in the horizontal $y z$ plane corresponding to $\phi=\pi / 2$. For this situation $\left|S_{1}(\theta)\right|^{2}$ for plane-wave incidence is shown in Fig. 3. Similarly, $\left|S_{1}(\theta, \pi / 2)\right|^{2}$ for Gaussian beam incidence with $x_{0}=0, z_{0}=0$, and $y_{0}$ equal to $-40,0$, and $40 \mu \mathrm{m}$ is shown in Figs. 4(a), 4(b), and 4(c), respectively. Since the beamwidth is approximately half of the particle diameter, Figs. 4(a)-4(c) exhibit quite different behaviors, and since $2 \pi a / \lambda=528.8$ for this particle, the features of the far-field scattered intensity are expected to be qualitatively understood on the basis of the interaction of geometrical light rays with the particle. For plane-wave incidence, the important ray trajectories are shown in Fig. 5. The diffracted rays and the transmitted rays $(p=1)$ dominate near-forward scattering, the specularly reflected rays $(p=0)$ dominate the scattering for $\theta \approx$ $90^{\circ}$, and the rays that emerge from the particle after one or two internal reflections ( $p=2,3$, respectively) produce rainbows that dominate the scattering in the backward hemisphere. Similarly, for Gaussian beam incidence with $x_{0}=z_{0}=0$ and $y_{0}=-40,0$, and $40 \mu \mathrm{m}$, the dominant ray trajectories are shown in Figs. 6(a), 6(b), and 6(c), respectively. For $y_{0}=-40 \mu \mathrm{m}$, only the $p=1,2$ rays provide strong contributions since the $p=0,3$ rays leave the particle on the opposite side. For $y_{0}=0 \mu \mathrm{m}$, only paraxial rays strike the particle, giving $p=1$ transmission dominance in the forward hemisphere and $p=0$ reflection dominance in the backward hemisphere. No rainbows are expected to appear since all rainbows require rays that are incident near the edges of the particle. For $y_{0}=40 \mu \mathrm{m}$,

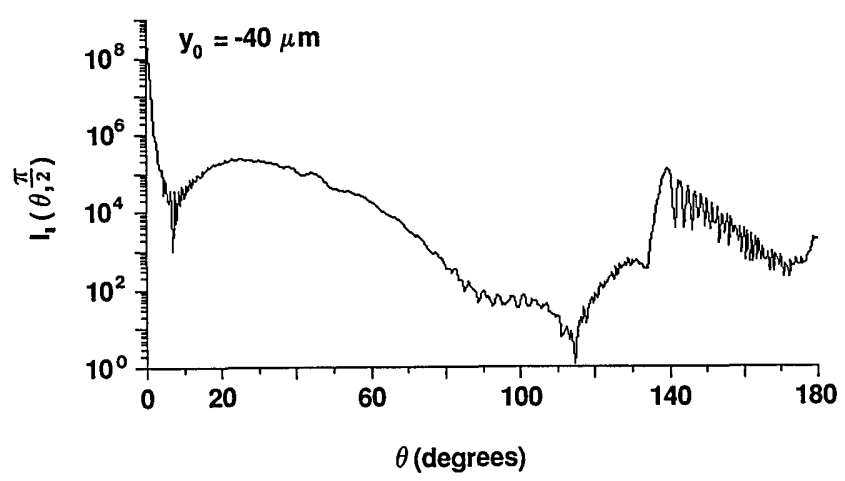

(a)

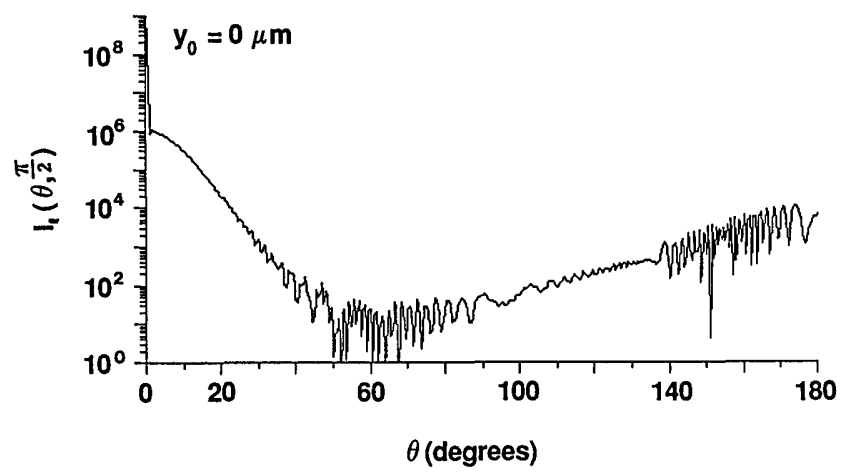

(b)

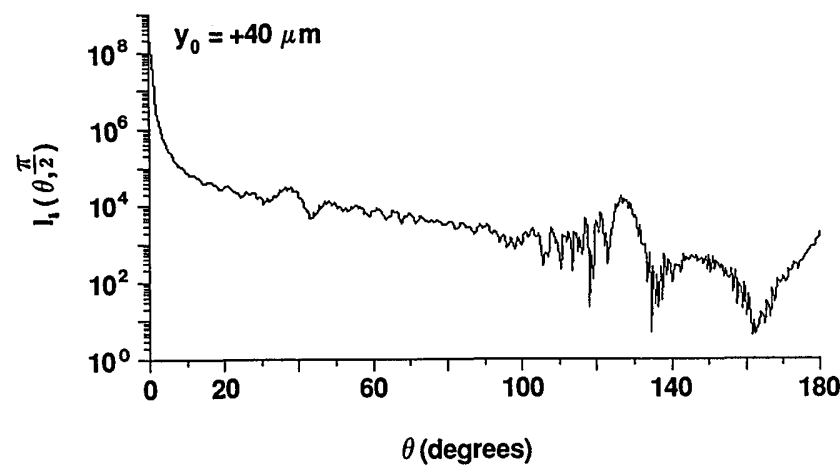

(c)

Fig. 4. Far-field scattered intensity $I_{1}(\theta, \pi / 2)=\left|S_{1}(\theta, \pi / 2)\right|^{2}$ as a function of scattering angle for a Davis first-order Gaussian laser beam with $\lambda=0.5145 \mu \mathrm{m}$ and $w=20 \mu \mathrm{m}$ and polarized in the $x$ direction incident upon a spherical particle with $a=43.3 \mu \mathrm{m}$ and $n=1.33$. The center of the focal waist of the beam is located at (a) $x_{0}=z_{0}=0, y_{0}=-40 \mu \mathrm{m}$; (b) $x_{0}=z_{0}=0, y_{0}=0 \mu \mathrm{m}$; and (c) $x_{0}=z_{0}=0, y_{0}=40 \mu \mathrm{m}$. The center of the particle is at the origin of coordinates. 


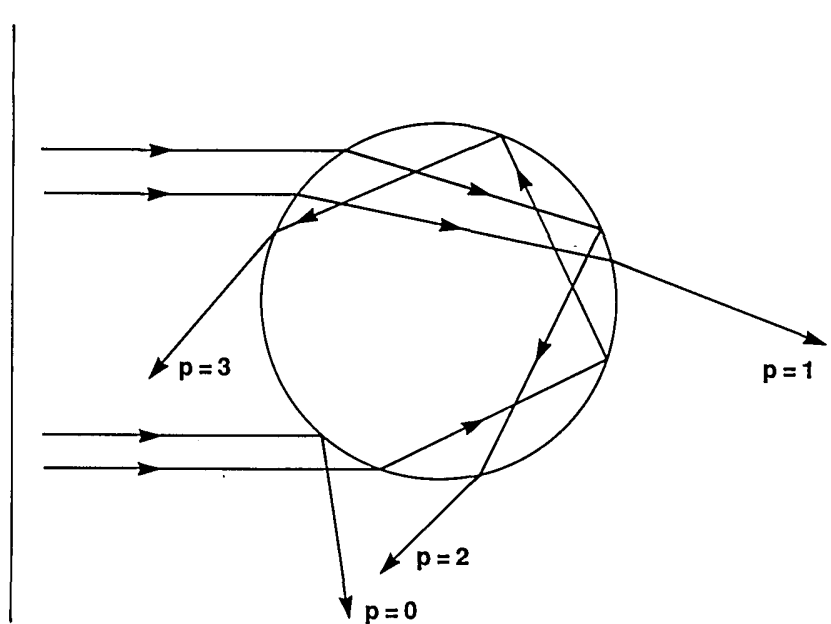

Fig. 5. Dominant ray trajectories for plane-wave incidence are specular reflection $(p=0)$, transmission $(p=1)$, transmission after one internal reflection $(p=2)$, and transmission after two internal reflections $(p=3)$.

only the $p=0,3$ rays are expected to dominate because the $p=1,2$ rays emerge from the particle on the opposite side.

We tested these geometric optics predictions by performing a Debye-series decomposition of the partial-wave scattering amplitudes for both plane-wave and Gaussian beam incidence. The Debye-series decomposition of the plane-wave partial-wave scattering amplitudes may be written as

$$
\left.\begin{array}{l}
a_{l} \\
b_{l}
\end{array}\right\}=\frac{1}{2}\left[1-R_{l}^{22}-\sum_{p=1}^{\infty} T_{l}^{21}\left(R_{l}^{11}\right)^{p-1} T_{l}^{12}\right],
$$

where the first term represents the diffraction of the spherical multipole partial waves, the second term represents their reflection from the surface of the particle, and the third term represents their penetration into the particle and subsequent exit after $p-1$ internal reflections. The expressions for $R_{l}{ }^{11}, R_{l}{ }^{22}, T_{l}{ }^{21}$, and $T_{l}{ }^{12}$ in terms of spherical Bessel and spherical Neumann functions are given elsewhere. ${ }^{25-29}$ Since the partial-wave scattering amplitudes $\alpha_{l m}$ and $\beta_{l m}$ for Gaussian beam incidence have the product decomposition of Eqs. (14), the Debye-series analysis of both plane-wave incidence and Gaussian beam incidence proceeds identically.

The individual Debye-series components for plane-wave incidence for $p \leq 12$ are given in Figs. 7(a) and 7(b). As expected from ray theory, the sum of diffraction plus $0 \leq$ $p \leq 3$ plus the interference among these terms almost exactly fits the full Mie scattering curve of Fig. 3. The rainbows for $4 \leq p \leq 10$ and $p=12$ do not visibly contribute to the scattered intensity because of the greatly decreased amplitude of the spherical multipole waves after many internal reflections. An exception is provided by the $p=11$ rainbow that occurs near $\theta=\pi$. Its contribution to $I(\theta)$ is amplified because of the axial focusing characteristic of glory scattering. ${ }^{41-43}$

The individual Debye-series components for $\left|S_{1}(\theta, \pi / 2)\right|^{2}$ for Gaussian beam incidence with $x_{0}=z_{0}=0$ and $y_{0}$ equal to $-40,0$, and $40 \mu \mathrm{m}$ are given in Figs. 8(a), 8(b), and $8(\mathrm{c})$, respectively. Since the width of the Gaussian beam is smaller than the diameter of the particle, many of the physical mechanisms that produce strong scattering for plane-wave incidence are greatly reduced in strength, permitting the otherwise much weaker physical mechanisms (i.e., high-order rainbows) to influence the behavior of the scattered intensity. For example, in Fig. 8(a) the reductions in $p=0$ and $p=3$ permit the $p=6$ and $p=10$ rainbows to dominate the scattering for $\theta \approx 130^{\circ}$ and $\theta \approx 100^{\circ}$, respectively. In Fig. 8 (c) the reductions in $p=1$ and $p=2$ permit the $p=4,7,11$ rainbows to dominate the scattering for $\theta$ approximately equal to $40^{\circ}, 140^{\circ}$ and $180^{\circ}$, respectively. For much smaller droplets, e.g., $a=5 \mu \mathrm{m}$, rainbow formation is strongly suppressed, and the features of the $I(\theta, \phi)$ graphs for Gaussian beam incidence do not have this interpretation.

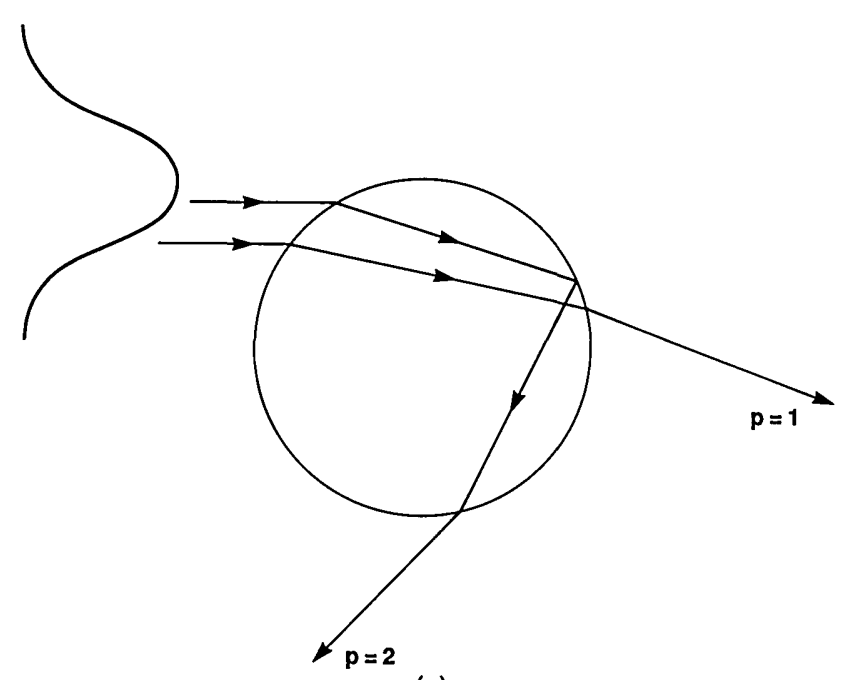

(a)

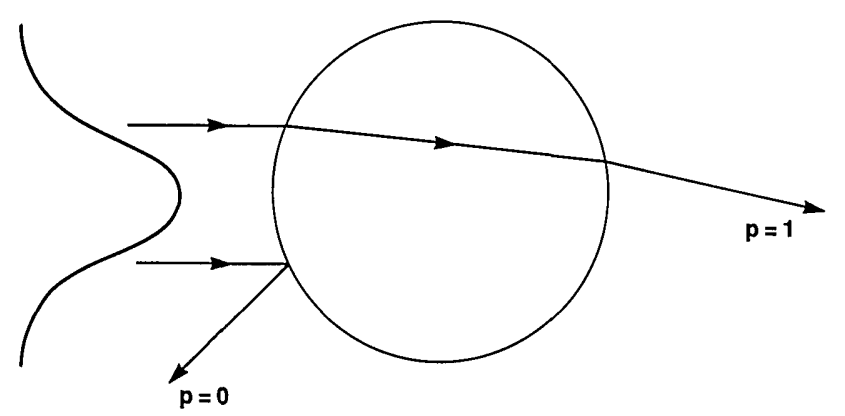

(b)

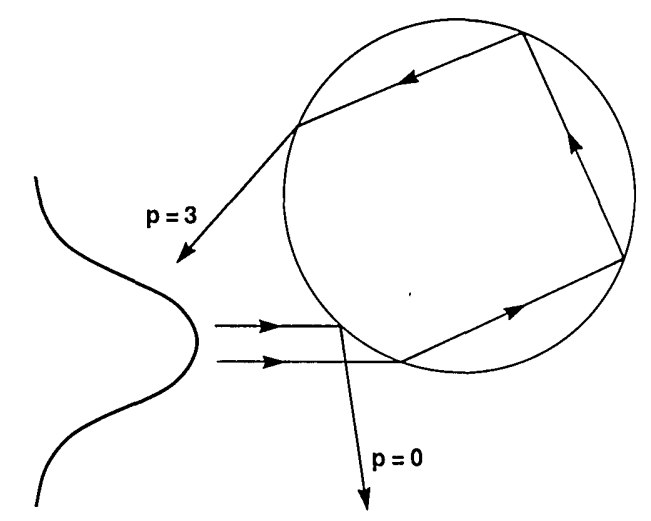

(c)

Fig. 6. Dominant ray trajectories for Gaussian beam incidence for $w \ll a$ are (a) $p=1,2$ for $y_{0}<0$; (b) $p=0,1$ for $y_{0} \approx 0$; and (c) $p=0,3$ for $y_{0}>0$. 


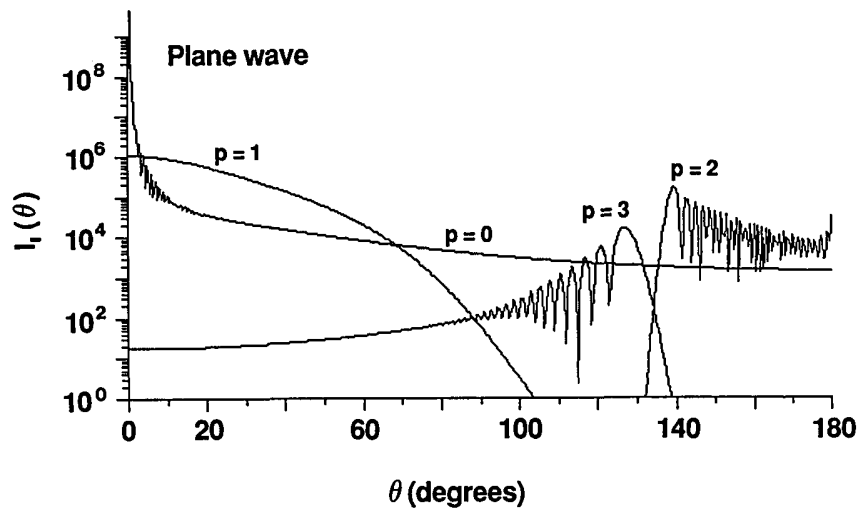

(a)

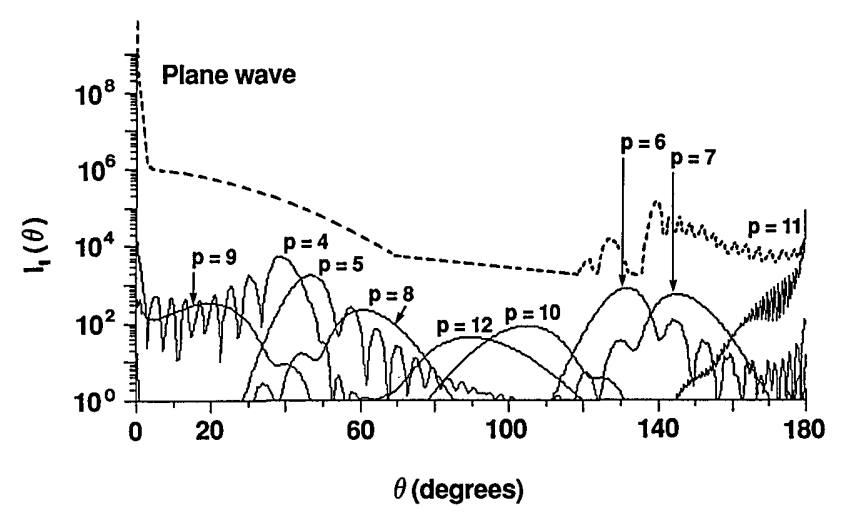

(b)

Fig. 7. Debye-series contributions to $I_{1}(\theta)=\left|S_{1}(\theta)\right|^{2}$ for planewave incidence with the polarization in the $x$ direction, $\lambda=$ $0.5145 \mu \mathrm{m}, a=43.3 \mu \mathrm{m}$, and $n=1.33$. (a) The sum of the contributions that are due to diffraction plus $p=0,1,2,3$ almost exactly fit the full Mie intensity of Fig. 3. (b) The contributions that are due to the $4 \leq p \leq 12$ rainbows are weak compared with the contributions of $p \leq 3$. The dashed curve represents the sum of $0 \leq p \leq 3$ from Fig. 7(a).

For plane-wave incidence, the presence of high-order rainbows was evident only in the Fourier transform of the scattered intensity, ${ }^{27,44,45}$ which corresponds to the glare spot observations reported in Refs. 19, 20, and 46. This was true because the high-order rainbows were obscured by the reflected and transmitted light and because the Fourier transform separates overlapping signals of different spatial frequencies. For Gaussian beam incidence with $w<a$, the presence of certain high-order rainbows should be evident in the scattered intensity itself since the scattering processes are now channeled to either leftof-center or right-of-center scattering angles, again separating the formerly overlapping signals of plane-wave incidence. This physical interpretation also explains the results of Ref. 47 for Gaussian beam incidence upon an absorbing sphere. The absorption extinguishes the $p \geq 1$ Debye terms corresponding to waves that are transmitted through the sphere, leaving only diffraction and specular reflection to contribute to far-field scattering.

\section{OBSERVATION OF RAINBOWS PRODUCED BY A GAUSSIAN BEAM}

An experiment was performed to observe the dependence of the strength of the different-order rainbows on the po- sitioning of an incident Gaussian laser beam when the beam width was much smaller than the diameter of the droplet. The experimental apparatus is described in detail in Ref. 29. Briefly, the polarized $\lambda=0.5145-\mu \mathrm{m}$ beam of a $5-W$ argon-ion laser was focused by a $25-\mathrm{cm}$ focal-length lens. The waist of the focused beam was measured to be $2 w=40 \pm 5 \mu \mathrm{m}$. This focused beam was incident upon falling water droplets produced by a vibrating orifice generator. For the two experimental runs, the diameter of the droplets was measured to be $2 a=47.8 \pm$ $1.5 \mu \mathrm{m}$ and $2 a=86.6 \pm 1.5 \mu \mathrm{m}$. In the first case, the beam waist was $\sim 84 \%$ of the particle diameter, and in the

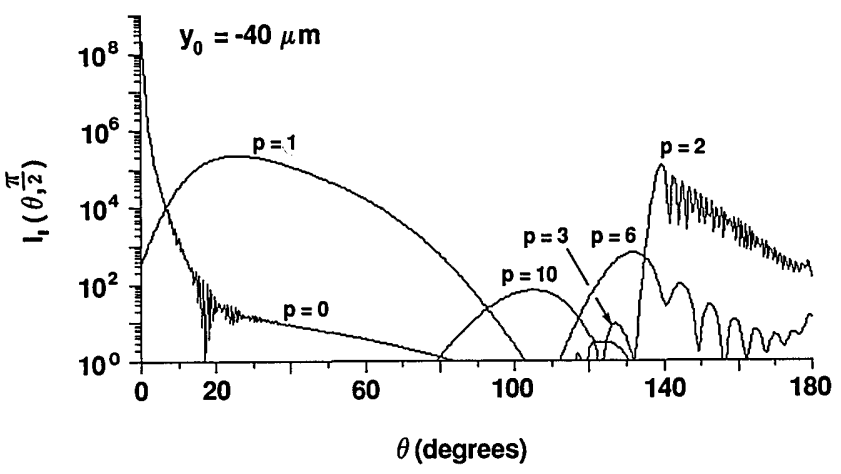

(a)

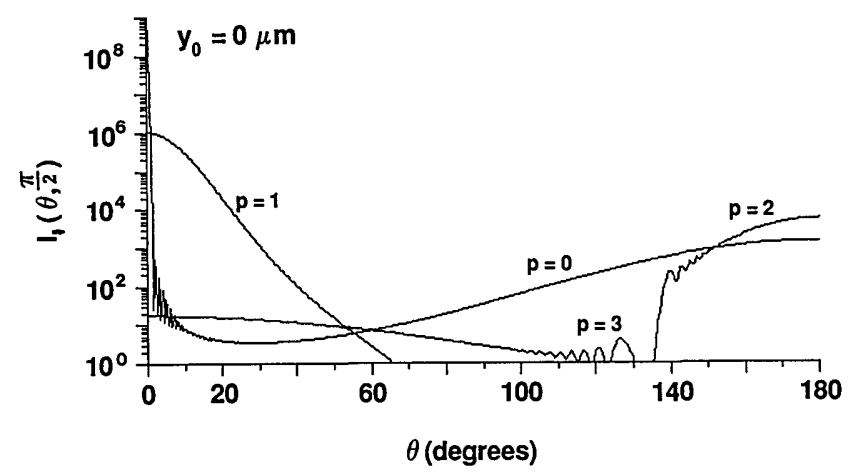

(b)

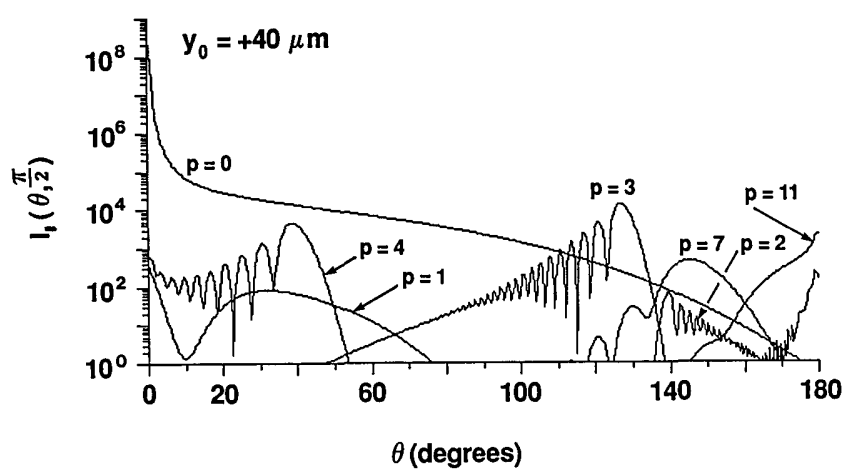

(c)

Fig. 8. Debye-series contributions to $I_{1}(\theta, \pi / 2)=\left|S_{1}(\theta, \pi / 2)\right|^{2}$ for a Davis first-order Gaussian beam incident with the polarization in the $x$ direction, $\lambda=0.5145 \mu \mathrm{m}, w=20 \mu \mathrm{m}, a=43.3 \mu \mathrm{m}$, and $n=1.33$. (a) For $y_{0}=-40.0 \mu \mathrm{m}$, the sum of the contributions that are due to diffraction plus $p=0,1,2,6,10$ almost exactly fit the full wave-theory intensity of Fig. 4(a). (b) For $y_{0}=0 \mu \mathrm{m}$, the sum of the contributions that are due to diffraction plus $p=0,1,2$ almost exactly fit the full wave-theory intensity of Fig. 4(b). (c) For $y_{0}=40.0 \mu \mathrm{m}$, the sum of the contributions that are due to diffraction plus $p=0,3,7,11$ almost exactly fit the full wavetheory intensity of Fig. 4(c). 


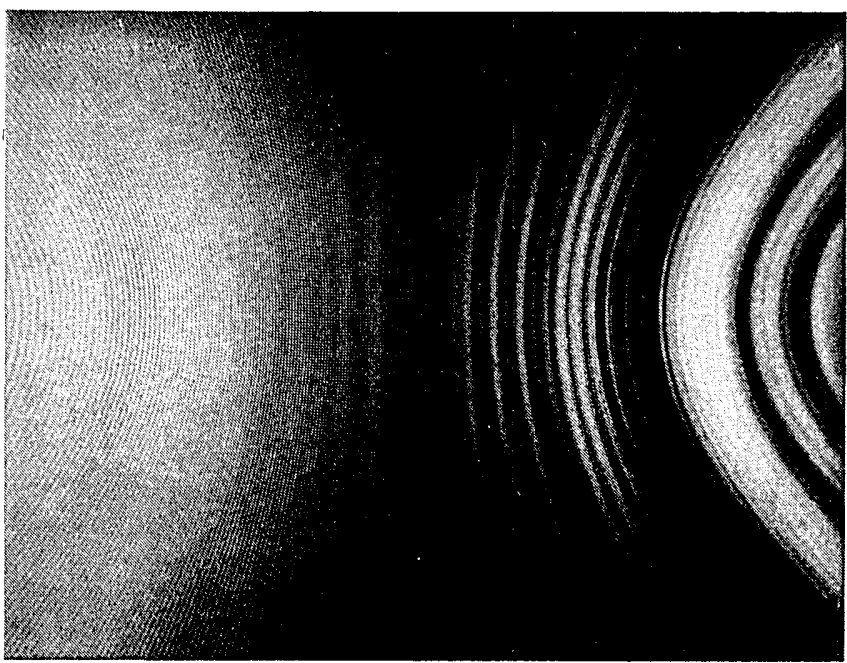

(a)

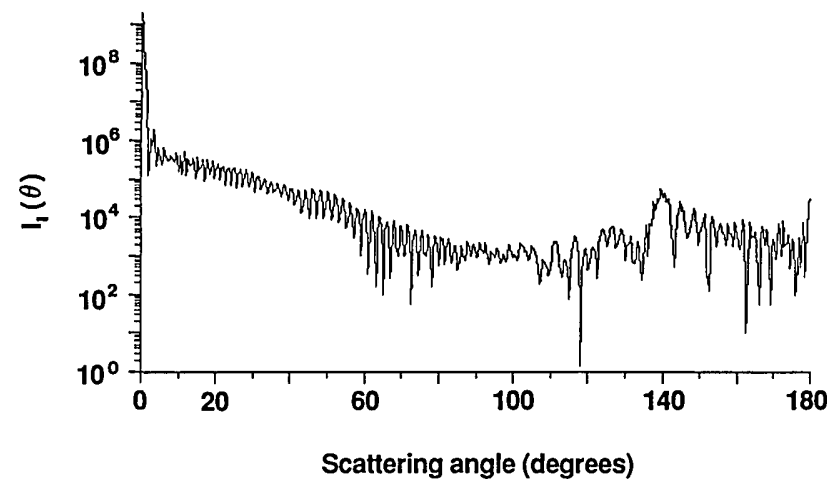

(b)

Fig. 9. (a) Experimental intensity spectrum for $30^{\circ} \leq \theta \leq 150^{\circ}$ (left to right) for a plane wave that is polarized in the $x$ direction and with $\lambda=0.5145 \mu \mathrm{m}$ incident upon a spherical water droplet with $a \approx 23.9 \mu \mathrm{m}$. The reflection-transmission interference is on the left-hand side of the photograph, and the second- and firstorder rainbows along with their first few supernumeraries are on the right-hand side. (b) $I_{1}(\theta)=\left|S_{1}(\theta)\right|^{2}$ for plane-wave incidence with the same parameters as in the experiment whose results are shown in (a).

second case it was $\sim 46 \%$ of the diameter. The light that was scattered into the angular region $70^{\circ}<\phi<110^{\circ}$ and $30^{\circ}<\theta<150^{\circ}$ exposed a strip of Polaroid film that was positioned a number of centimeters away.

As a test of this method, a minimally focused laser beam (i.e., $w \approx 500 \mu \mathrm{m}$ ) was incident upon the droplets. The resulting scattered intensity is shown in Fig. 9(a). Since the minimally focused beam with $w \gg a$ approximates a plane wave, Fig. 9(a) should be compared with the theoretical intensity $I_{1}(\theta)$ for plane-wave incidence with the same particle and beam parameters given in Fig. 9(b). The comparison between theory and experiment is qualitatively quite good. The bright, fine periodicity interference pattern on the left-hand side of Fig. 9(a) is the reflection-transmission interference pattern $[p=$ $0+1$; see Fig. 7(a)]. The scattering is weakest near the center of Fig. 9(a) at $\theta \approx 100^{\circ}$ corresponding to specular reflection. On the right-hand side of Fig. 9(a) are the second-order rainbow ( $p=3$ ) with its first three supernumeraries to its left and the first-order rainbow $(p=2)$ with its first two supernumeraries to its right. Between them is Alexander's dark band, in which only specular re- flection contributes. All these features are faithfully reproduced in the theoretical intensity of Fig. 9(b).

The experimental intensity for $a \approx 23.9 \mu \mathrm{m}$ and $x_{0}=$ $z_{0}=0, y_{0} \approx-22 \mu \mathrm{m}$ is shown in Fig. 10(a), and the corresponding theoretical intensity $I_{1}(\theta, \pi / 2)$ for Gaussian beam incidence is given in Fig. 10(d). Again the agreement between theory and experiment is qualitatively quite good. The broad bright region on the left-hand side of Fig. 10(a) is the $p=1$ transmitted rays. Since the critical scattering angle for transmission ${ }^{29}$ is $\theta_{c}=82.8^{\circ}$, the sharp cutoff of the transmitted light for $\theta \approx \theta_{c}$ is readily evident in Fig. 10(a). The first-order rainbow $(p=2)$ and its first two supernumeraries appear prominently on the righthand side of Fig. 10(a). The second-order rainbow and the specularly reflected light have been channeled to the other side of the droplet by the placement of the incident laser beam. According to Fig. 8(a), the fifth- and ninthorder rainbows ( $p=6,10$, respectively) should appear weakly in the broad dark region between the transmission cutoff and the first-order rainbow. Neither appears in Fig. 10(a), and it is uncertain whether exposing the film for much longer periods of time would have unambiguously showed them without producing bleedover from the adjacent overexposed regions.

The experimental intensity for $a \approx 23.9 \mu \mathrm{m}$ and $x_{0}=$ $z_{0}=0$, and $y_{0} \approx 7 \mu \mathrm{m}$ is shown in Fig. $10(\mathrm{c})$, and the corresponding theoretical intensity $I_{1}(\theta, \pi / 2)$ for Gaussian beam incidence is given in Fig. 10(e). For this case, the scattered-light level is much weaker and is relatively devoid of interesting features. The paraxial beam incidence has produced neither the first- nor the secondorder rainbow. There is a single broad dim fringe extending for $90^{\circ}<\theta<100^{\circ}$ with finer interference patterns to each side. These details are also evident in Fig. 10(e), which again shows the average light level to be nearly constant for $\theta>40^{\circ}$. Referring to Fig. 8(b), this largely featureless pattern is probably the result of weak reflection-transmission interference for $\theta<90^{\circ}$ and weak reflection-one- or two-internal-reflection interference for $\theta>100^{\circ}$.

The experimental intensity for $a \approx 43.3 \mu \mathrm{m}$ and $x_{0}=$ $z_{0}=0, y_{0} \approx 40 \mu \mathrm{m}$ is shown in Fig. $10(\mathrm{~b})$. The corresponding theoretical intensity $I_{1}(\theta, \pi / 2)$ for Gaussian beam incidence is given in Fig. 4(c). Again the comparison between theory and experiment is quite good, although more supernumeraries of the second-order rainbow are evident in Fig. 10(b) than can be easily identified in Fig. 4(c). Decreasing the beam width to $w=15 \mu \mathrm{m}$ in the theoretical intensity improved the agreement. The broad bright region on the left-hand side of Fig. 10(b) is the specularly reflected light $(p=0)$. As is shown in Fig. 8(c), it extends all the way out to the second-order rainbow supernumeraries and does not have a sharp cutoff as did the transmitted light in Figs. 10(a) and 10(d). On the righthand side of Fig. 10(e) is the second-order rainbow $(p=3)$, its supernumeraries to its left, and a single broad dim fringe to the right. Again referring to Fig. 8(c), I tentatively identify the single broad fringe with the sixthorder rainbow $(p=7)$ rather than the first-order rainbow $(p=2)$ for two reasons: first, the placement of the incident Gaussian beam should have decreased the intensity of the $p=2$ rainbow to $\sim 10 \%$ of the intensity of the $p=7$ rainbow, and second, the first-order rainbow peak should be narrower than the second-order rainbow peak, whereas 


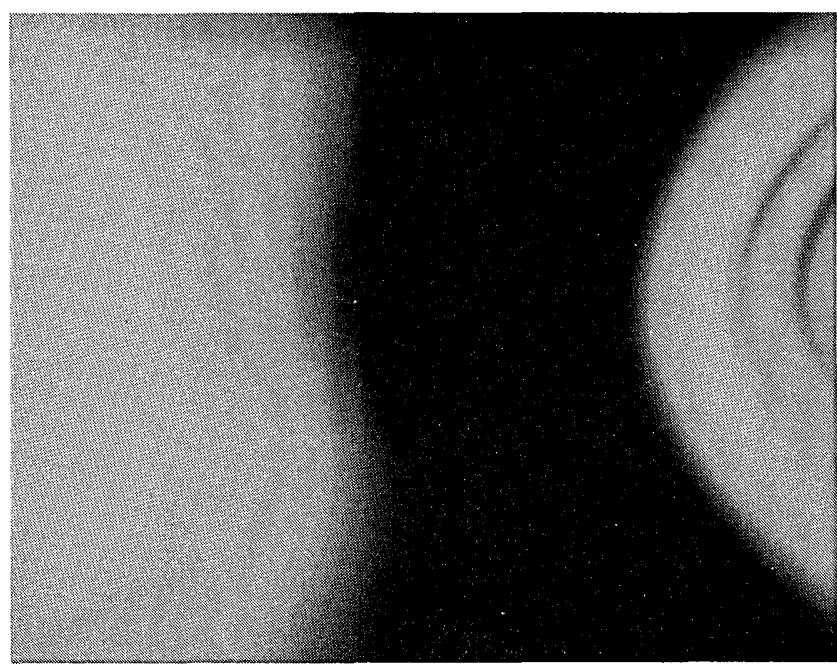

(a)

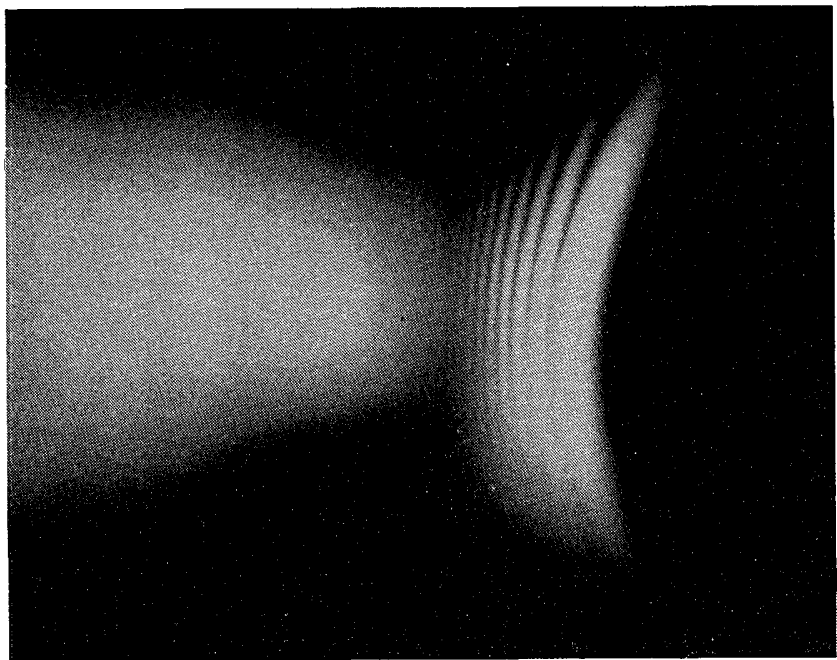

(b)

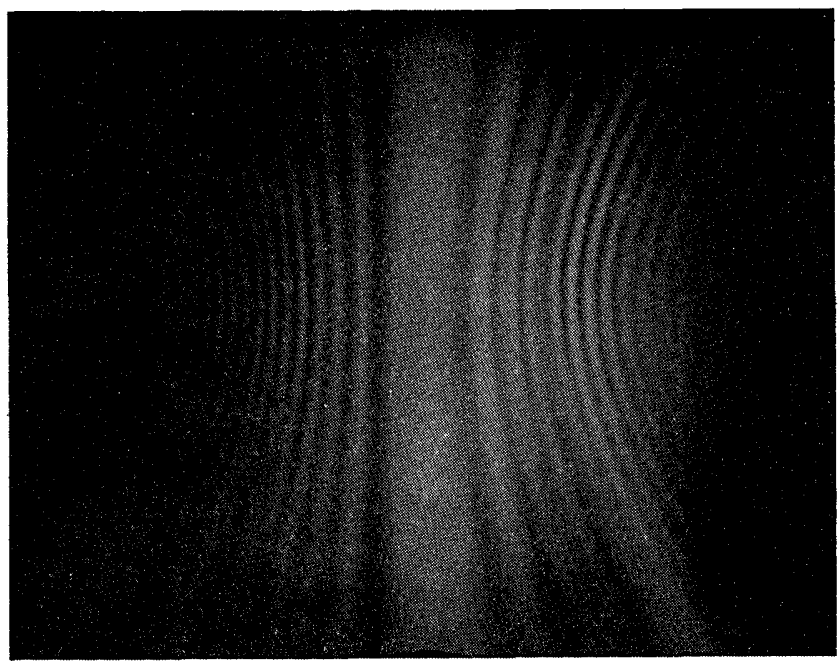

(c)

in Fig. 10(b) the single dim broad fringe is approximately twice as wide as the second rainbow peak. Thus I believe that I have observed a high-order rainbow in the light that was scattered from an incident Gaussian beam by a spherical water droplet.

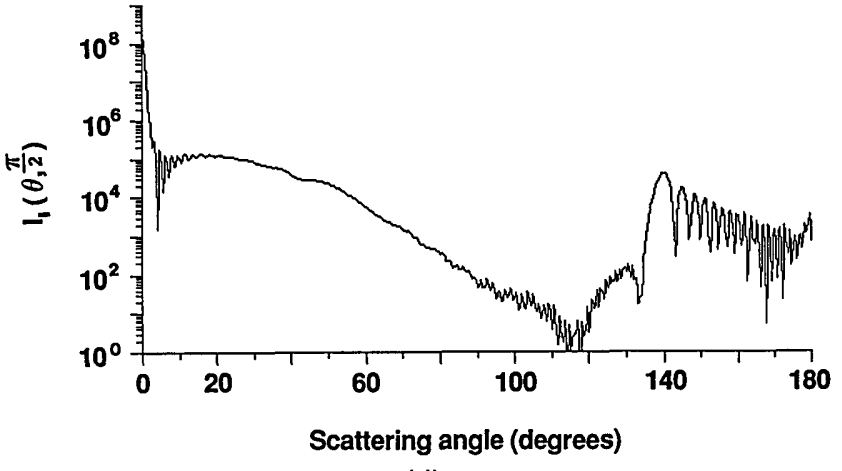

(d)

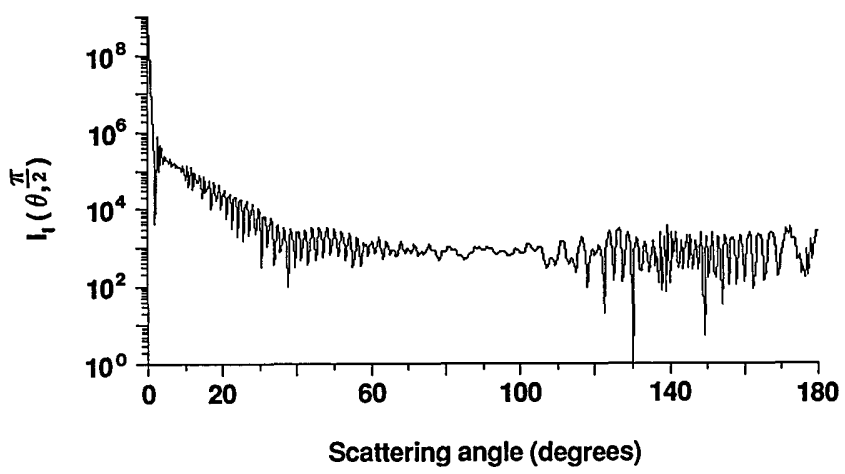

(e)
Fig. 10. (a) Experimental intensity spectra for $30^{\circ} \leq \theta \leq 150^{\circ}$ (left to right) for a focused Gaussian laser beam polarized in the $x$ direction and with $\lambda=0.5145 \mu \mathrm{m}$ and $w \approx 20 \mu \mathrm{m}$ incident upon a spherical water droplet with $a=23.9 \mu \mathrm{m}$ and $y_{0} \approx-22 \mu \mathrm{m}$. The transmitted light is on the left-hand side of the photograph, and the first-order rainbow is on the right-hand side. (b) The experimental intensity for $a \approx 43.3 \mu \mathrm{m}$ and $y_{0} \approx 40 \mu \mathrm{m}$. The specularly reflected light is on the left-hand side of the photograph, and the second-order rainbow is on the right-hand side. The single wide dim fringe to the right of the main second-order rainbow fringe is tentatively identified as the main fringe of the sixth-order rainbow. Compare this photograph with Fig. 4(c). (c) The experimental intensity for $a \approx 23.9 \mu \mathrm{m}$ and $y_{0} \approx 7 \mu \mathrm{m}$. The scattered light is much dimmer, and both the first- and second-order rainbows are absent. (d) $I_{1}(\theta, \pi / 2)=\left|S_{1}(\theta, \pi / 2)\right|^{2}$ with the same parameters as in the experiment whose result is shown in (a). (e) $I_{1}(\theta, \pi / 2)=\left|S_{1}(\theta, \pi / 2)\right|^{2}$ with the same parameters as in the experiment whose result is shown in (c).

\section{CONCLUSION}

The ubiquity of experiments that scatter small-diameter laser beams off millimeter-sized raindrops or that scatter focused laser beams off small water droplets produced 
by vibrating orifice generators necessitates a theory for Gaussian beam scattering and fast-running computer programs to implement it. The theory of Gaussian beam scattering as developed by Gouesbet et al. and Barton et al. may be straightforwardly and rapidly computed and is thus expected to be of great use in a variety of experimental situations. The notation that I employed in Section 2 is a hybrid of their notations. I have chosen what I believe are the best features of each of their formalisms in constructing my own. Computer programs for calculating Gaussian beam scattering already exist. However, in Section 3 I have given what I believe is a fuller account of the numerical details that are involved in constructing such programs than exists elsewhere in the literature. Similarly, the far-field scattered intensities that I calculated in Section 4 have also appeared elsewhere. However, I have given a detailed physical motivation for the novel features of these $I(\theta, \phi)$ spectra, namely, that they arise from the contributions of high-order rainbows and a simultaneous suppression of a number of the mechanisms that dominate for plane-wave incidence. In Section 5 I have given tentative evidence that these high-order rainbow contributions do in fact occur.

\section{APPENDIX A: DERIVATION OF THE LOCALIZATION APPROXIMATION FOR AN ON-AXIS GAUSSIAN BEAM}

For a Davis first-order Gaussian beam on axis, the radial component of the incident fields is given by Eqs. (24), and the incident beam partial-wave coefficient $I_{l}$ is given by Eq. (26). Consider first the special case $f(k r, \theta)=1$. Using the partial-wave decomposition of the scalar plane wave $^{48}$ and the identity

$$
\sin (\theta) P_{l}^{1}[\cos (\theta)]=\frac{l(l+1)}{2 l+1}\left\{P_{l-1}[\cos (\theta)]-P_{l+1}[\cos (\theta)]\right\}
$$

we obtain

$$
\begin{aligned}
\int_{0}^{\pi} \sin (\theta) \mathrm{d} \theta P_{l}[\cos (\theta)] \exp [i k r \cos (\theta)]= & 2 i^{l} j_{l}(k r), \\
\int_{0}^{\pi} \sin ^{2}(\theta) \mathrm{d} \theta P_{l}^{1}[\cos (\theta)] \exp [i k r \cos (\theta)]= & 2 i^{l-1} l(l+1) \\
& \times j_{l}(k r) / k r .
\end{aligned}
$$

Consider now the integral

$$
J_{l}=\int_{0}^{\pi} \sin ^{2}(\theta) \mathrm{d} \theta P_{l}^{1}[\cos (\theta)] f(k r, \theta) \exp [i k r \cos (\theta)],
$$

where $f(k r, \theta)$ is slowly varying. Such is the case for the Davis first-order beam of Eqs. (19)-(22), where

$$
\begin{aligned}
f(k r, \theta)= & \frac{1}{D} \exp \left[\frac{-k^{2} r^{2} \sin ^{2}(\theta) / k^{2} w^{2}}{D}\right] \\
& \times\left[1-\frac{2 i s k r \cos (\theta) / k w}{D}\right] \exp \left(-i k z_{0}\right), \\
D= & 1+\frac{2 i\left[k r \cos (\theta)-k z_{0}\right] s}{k w}
\end{aligned}
$$

The $\sin ^{2}(\theta)$ factor in the integrand ensures that the intervals near $\theta=0, \pi$ do not contribute to the integral. Thus, for $l \gg 1, P_{l}{ }^{1}[\cos (\theta)]$ may be replaced by relation (45), and

$$
\begin{aligned}
J_{l} \approx & \int_{0}^{\pi} \sin ^{2}(\theta) \mathrm{d} \theta\left[\frac{2 l}{\pi \sin (\theta)}\right]^{1 / 2} \sin \left[\left(l+\frac{1}{2}\right) \theta-\frac{\pi}{4}\right] \\
& \times f(k r, \theta) \exp [i k r \cos (\theta)] .
\end{aligned}
$$

By use of the substitution

$$
\alpha=\theta-\frac{\pi}{2}
$$

to change to a symmetric integration interval, this becomes

$$
\begin{aligned}
J_{l}= & \int_{-\pi / 2}^{\pi / 2} \cos ^{2}(\alpha) \mathrm{d} \alpha\left[\frac{2 l}{\pi \cos (\alpha)}\right]^{1 / 2} \sin \left[\left(l+\frac{1}{2}\right) \alpha+\frac{l \pi}{2}\right] \\
& \times f\left(k r, \alpha+\frac{\pi}{2}\right) \exp [-i k r \sin (\alpha)] \\
= & \frac{i^{l}}{2 i} \int_{-\pi / 2}^{\pi / 2} \cos ^{2}(\alpha) \mathrm{d} \alpha\left[\frac{2 l}{\pi \cos (\alpha)}\right]^{1 / 2} f\left(k r, \alpha+\frac{\pi}{2}\right) \\
& \times \exp \left[i\left(l+\frac{1}{2}\right) \alpha-i k r \sin (\alpha)\right]-\frac{(-i)^{l}}{2 i} \\
& \times \int_{-\pi / 2}^{\pi / 2} \cos ^{2}(\alpha) \mathrm{d} \alpha\left[\frac{2 l}{\pi \cos (\alpha)}\right]^{1 / 2} f\left(k r, \alpha+\frac{\pi}{2}\right) \\
& \times \exp \left[-i\left(l+\frac{1}{2}\right) \alpha-i k r \sin (\alpha)\right] .
\end{aligned}
$$

The interval near $\alpha=0$ provides the largest contribution to the integral. For this situation the integrand of second integral in Eq. (A9) is always rapidly varying, thus contributing little to the value of $J_{l}$. However, the complex exponential in the integrand of the first integral has a stationary point when $\alpha$ is zero and $k r=l+(1 / 2)$. Thus the value of $J_{l}$ is well approximated if the slowly varying factor $f$ is evaluated at the stationary point

$$
k r=l+\frac{1}{2}, \quad \theta=\frac{\pi}{2}
$$

or

$$
k r \cos (\theta) \rightarrow 0, \quad k r \sin (\theta) \rightarrow l+\frac{1}{2}
$$

and brought outside the integral. We thus obtain

$$
\begin{aligned}
J_{l} & \approx f\left(l+\frac{1}{2}, \frac{\pi}{2}\right) \int_{0}^{\pi} \sin ^{2}(\theta) \mathrm{d} \theta P_{l}{ }^{1}[\cos (\theta)] \exp [i k r \cos (\theta)] \\
& =f\left(l+\frac{1}{2}, \frac{\pi}{2}\right) 2 i^{l-1} l(l+1) j_{l}(k r) / k r
\end{aligned}
$$

The incident beam partial-wave coefficient $I_{l}$ is then

$$
I_{l} \approx f\left(l+\frac{1}{2}, \frac{\pi}{2}\right),
$$

which for the incident beam function of Eq. (A5) gives relation (50). This argument is analogous to the stationaryphase derivation of van de Hulst's localization principle, which associates a geometrical light ray with the small group of partial waves for which the phase is stationary. 
For the Davis first-order beam, we found relation (A13) to be within 1 part in $10^{5}$ of the value of $I_{l}$ by using direct integration with $r=a$. For the function

$$
f(k r, \theta)=\exp \left[\frac{-k^{2} r^{2} \sin ^{2}(\theta) / k^{2} w^{2}}{|D|^{2}}\right],
$$

which is also slowly varying but is not an approximate solution to the Helmholtz equations, as was Eq. (A5), we found relation (A13) to be within only 1 part in $10^{2}$ of the value of $I_{l}$ by using direct integration with $r=a$. It appears that the closer $f(k r, \theta)$ in Eqs. (24) is to a solution of the Helmholtz equation, the better the localization approximation works. I conjecture that there may well be a way to prove the localization approximation by using the properties of functions $f(k r, \theta)$, whose scalar radiation potential satisfies Eq. (3).

This conjecture would be of special importance for off-axis incidence since the stationary-phase method of relations (A1)-(A13) fails for this situation. For example, for the Davis first-order beam off axis, following Ref. 16, we have

$$
\begin{aligned}
E_{\text {inc }}^{\mathrm{rad}}(r, \theta, \phi)= & {\left.\left[F \cos (\phi)+G x_{0}\right)\right] } \\
& \times \exp \left\{2 r \sin (\theta)\left[x_{0} \cos (\phi)+y_{0} \sin (\phi)\right] / w^{2} D\right\} \\
= & \frac{F}{2} \sum_{j=0}^{\infty} \sum_{p=0}^{j} \Psi_{j p} \exp [i(j-2 p+1) \phi] \\
& +\frac{F}{2} \sum_{j=0}^{\infty} \sum_{p=0}^{j} \Psi_{j p} \exp [i(j-2 p-1) \phi] \\
& +G x_{0} \sum_{j=0}^{\infty} \sum_{p=0}^{j} \Psi_{j p} \exp [i(j-2 p) \phi]
\end{aligned}
$$

where

$$
\begin{aligned}
F & =\psi_{00} \exp [i k r \cos (\theta)] \sin (\theta)\left[1-\frac{2 i s r \cos (\theta) / w}{D}\right], \\
G & =\psi_{00} \exp [i k r \cos (\theta)] \frac{2 i s \cos (\theta) / w}{D}, \\
\psi_{00} & =\frac{1}{D} \exp \left\{\frac{-\left[r^{2} \sin ^{2}(\theta)+x_{0}^{2}+y_{0}^{2}\right] / w^{2}}{D}\right\} \exp \left(-i k z_{0}\right), \\
\Psi_{j p} & =\frac{\left(x_{0}-i y_{0}\right)^{j-p}\left(x_{0}+i y_{0}\right)^{p}}{(j-p) ! p !}\left[\frac{r \sin (\theta) / w^{2}}{D}\right]^{j} .
\end{aligned}
$$

The $\phi$ integration in Eqs. (5) may be performed to give

$$
\begin{aligned}
A_{l m}= & (-i)^{l-1} \frac{k r}{j_{l}(k r)} \frac{(l-|m|) !}{(l+|m|) !} \int_{0}^{\pi} \sin (\theta) \mathrm{d} \theta P_{l}^{m}[\cos (\theta)] \\
& \times\left(\frac{F}{2} \sum_{j=0}^{\infty} \sum_{p=0}^{j} \Psi_{j p} \delta_{j-2 p+1, m}+\frac{F}{2} \sum_{j=0}^{\infty} \sum_{p=0}^{j} \Psi_{j p} \delta_{j-2 p-1, m}\right. \\
& \left.+G x_{0} \sum_{j=0}^{\infty} \sum_{p=0}^{j} \Psi_{j p} \delta_{j-2 p, m}\right) .
\end{aligned}
$$

The integrals in Eq. (A20) that are associated with the $F$ terms are of the form

$$
\int_{0}^{\pi} \sin ^{2}(\theta) \mathrm{d} \theta P_{l}^{m}[\cos (\theta)] f_{m}(k r, \theta) \exp [i k r \cos (\theta)],
$$

where $f_{m}(k r, \theta)$ is a slowly varying function. At this point one would like to evaluate the slowly varying factor in the integrand at the stationary point of relations (A10) and A(11) to obtain the expressions of relations (51)-(53), bring it outside the integral, and evaluate the remaining integral

$$
\int_{0}^{\pi} \sin ^{2}(\theta) \mathrm{d} \theta P_{l}^{m}[\cos (\theta)] \exp [i k r \cos (\theta)]
$$

analytically. This integral is proportional to spherical Bessel functions divided by $(k r)^{(m+1) / 2}$ for odd $m$ and is proportional to Bessel functions divided by $(k r)^{(m+2) / 2}$ for even $m$. These forms will not cancel the $k r / j_{l}(k r)$ factor in Eq. (A20), except for $m=1$. Thus a different derivation of the localization approximation for off-axis incidence must be sought.

\section{ACKNOWLEDGMENTS}

This research was supported by National Aeronautics and Space Administration grant NCC 3-204. We thank Edward Hovenac of Sverdrup Technology Inc. at NASALewis for providing the photographs for Figs. 9(a), 10(a), $10(\mathrm{~b})$, and 10(c). We also thank Scott Schaub of the Center for Electro-Optics at the University of Nebraska for his careful verification of the results of our Gaussianbeam-scattering program.

\section{REFERENCES}

1. W. D. Bachalo, "Method for measuring the size and velocity of spheres by dual-beam light-scatter interferometry," Appl. Opt. 19, 363-370 (1980).

2. W. D. Bachalo and M. J. Houser, "Phase/Doppler spray analyzer for simultaneous measurements of drop size and velocity distributions," Opt. Eng. 23, 583-590 (1984).

3. S. V. Sankar and W. D. Bachalo, "Response characteristics of the phase Doppler particle analyzer for sizing spherical particles larger than the light wavelength," Appl. Opt. 30, 14871496 (1991).

4. S. V. Sankar, B. J. Weber, D. Y. Kamemoto, and W. D. Bachalo, "Sizing fine particles with the phase Doppler interferometric technique," Appl. Opt. 30, 4914-4920 (1991).

5. G. Grehan, G. Gouesbet, A. Naqwi, and F. Durst, "Evaluation of the phase-Doppler system using generalized Lorenz-Mie theory," presented at the International Conference on Multiphase Flows, University of Tsukuba, Japan, 1991.

6. S. A. Schaub and D. R. Alexander, "Theoretical analysis of the effects of particle trajectory on the performance of a phase/ Doppler particle analyzer," Appl. Opt. (to be published).

7. G. Grehan and G. Gouesbet, "Optical levitation of a single particle to study the theory of the quasi-elastic scattering of light," Appl. Opt. 19, 2485-2487 (1980).

8. G. Gouesbet, G. Grehan, and B. Maheu, "Scattering of a Gaussian beam by a Mie scatter center using a Bromwich formalism," J. Opt. (Paris) 16, 83-93 (1985).

9. G. Gouesbet, B. Maheu, and G. Grehan, "Light scattering from a sphere arbitrarily located in a Gaussian beam, using a Bromwich formalism," J. Opt. Soc. Am. A 5, 1427-1443 (1988).

10. J. P. Barton, D. R. Alexander, and S. A. Schaub, "Internal and near-surface electromagnetic fields for a spherical particle irradiated by a focused laser beam," J. Appl. Phys. 64, 16321639 (1988).

11. J. P. Barton, D. R. Alexander, and S. A. Schaub, "Internal fields of a spherical particle illuminated by a tightly focused laser beam: focal point positioning effects at resonance," J. Appl. Phys. 65, 2900-2906 (1989).

12. J. P. Barton, D. R. Alexander, and S. A. Schaub, "Theoretical determination of net radiation force and torque for a spheri- 
cal particle illuminated by a focused laser beam," J. Appl. Phys. 66, 4594-4602 (1989).

13. G. Grehan, B. Maheu, and G. Gouesbet, "Scattering of laser beams by Mie scatter centers: numerical results using a localized approximation," Appl. Opt. 25, 3539-3548 (1986).

14. B. Maheu, G. Grehan, and G. Gouesbet, "Generalized LorenzMie theory: first exact values and comparisons with the localized approximation," Appl. Opt. 26, 23-25 (1987).

15. G. Gouesbet, G. Grehan, and B. Maheu, "Computations of the $g_{n}$ coefficients in the generalized Lorenz-Mie theory using three different methods," Appl. Opt. 27, 4874-4883 (1988).

16. G. Gouesbet, G. Grehan, and B. Maheu, "Localized interpretation to compute all the coefficients $g_{n}{ }^{m}$ in the generalized Lorenz-Mie theory," J. Opt. Soc. Am. A 7, 998-1007 (1990).

17. H. C. van de Hulst, Light Scattering by Small Particles (Dover, New York, 1981), Sec. 12.31.

18. G. Gouesbet, G. Grehan, and B. Maheu, "Generalized LorenzMie theory and applications to optical sizing," in Proceedings of the Second International Congress on Optical Particle Sizing, E. D. Hirleman, ed. (Arizona State U. Press, Tempe, Ariz., 1990) pp. 118-126.

19. J. D. Walker, "Multiple rainbows from single drops of water and other liquids," Am. J. Phys. 44, 421-433 (1976).

20. J. D. Walker, "How to create and observe a dozen rainbows in a single drop of water," Sci. Am. 237(1), 138-144 (1977).

21. J. D. Walker, "Mysteries of rainbows, notably their rare supernumerary arcs," Sci. Am. 242(6), 174-184 (1980).

22. T. S. Fahlen and H. C. Bryant, "Optical back scattering from single water droplets," J. Opt. Soc. Am. 58, 304-310 (1968).

23. Ref. 17, Sec. 13.11.

24. C. F. Bohren and D. R. Huffman, Absorption and Scattering of Light by Small Particles (Wiley, New York, 1983), Sec. 7.1.2.

25. S. I. Rubinow, "Scattering from a penetrable sphere at short wavelengths," Ann. Phys. (N.Y.) 14, 305-332 (1961).

26. H. M. Nussenzveig, "High-frequency scattering by a transparent sphere. I. Direction reflection and transmission," J. Math. Phys. 10, 82-124 (1969).

27. J. A. Lock, "Cooperative effects among partial waves in Mie scattering," J. Opt. Soc. Am. A 5, 2032-2044 (1988).

28. J. A. Lock and E. A. Hovenac, "Internal caustic structure of illuminated liquid droplets," J. Opt. Soc. Am. A 8, 1541-1552 (1991).

29. E. A. Hovenac and J. A. Lock, "Assessing the contributions of surface waves and complex rays to far-field Mie scattering by use of the Debye series," J. Opt. Soc. Am. A 9, 781-795 (1992).
30. J. R. Reitz, F. J. Milford, and R. W. Christy, Foundations of Electromagnetic Theory, 3rd ed. (Addison-Wesley, Reading, Mass., 1979), Sec. 17.5

31. G. Arfken, Mathematical Methods for Physicists, 3rd ed. (Academic, New York, 1985), Eq. (11.175).

32. Ref. 31, Sec. 12.5 .

33. Ref. 9, Eq. (66) and Ref. 15, Eq. (19). See also Ref. 31, Eq. (11.174)

34. L. W. Davis, "Theory of electromagnetic beams," Phys. Rev. A 19, 1177-1179 (1979).

35. J. P. Barton and D. R. Alexander, "Fifth-order corrected electromagnetic field components for a fundamental Gaussian beam," J. Appl. Phys. 66, 2800-2802 (1989).

36. S. A. Schaub, J. P. Barton, and D. R. Alexander, "Simplified scattering coefficient expression for a spherical particle located on the propagation axis of a fifth-order Gaussian beam," Appl. Phys. Lett. 55, 2709-2711 (1989).

37. J. V. Dave, "Scattering of visible light by large water spheres," Appl. Opt. 8, 155-164 (1969).

38. W. J. Wiscombe, "Improved Mie scattering algorithms," Appl. Opt. 19, 1505-1509 (1980).

39. Ref. 24, Sec. 4.8 and App. A.

40. B. Maheu, G. Grehan, and G. Gouesbet, "Ray localization in Gaussian beams," Opt. Commun. 70, 259-262 (1989).

41. V. Khare and H. M. Nussenzveig, "Theory of the glory," Phys. Rev. Lett. 38, 1279-1282 (1977).

42. H. M. Nussenzveig, "Complex angular momentum theory of the rainbow and the glory," J. Opt. Soc. Am. 69, 1068-1079, 1193, 1194 (1979).

43. D. S. Langley and M. J. Morrell, "Rainbow-enhanced forward and backward glory scattering," Appl. Opt. 30, 3459-3467 (1991).

44. J. A. Lock, "Theory of the observations made of high-order rainbows from a single water droplet," Appl. Opt. 26, 52915298 (1987).

45. H. C. van de Hulst and R. T. Wang, "Glare points," Appl. Opt. 30, 4755-4763 (1991).

46. J. A. Lock and J. R. Woodruff, "Non-Debye enhancements in the Mie scattering of light from a single water droplet," Appl. Opt. 28, 523-529 (1989).

47. G. Grehan, B. Maheu, and G. Gouesbet, "Diffusion de la lumiere par une sphere dans le cas d'un faisceau d'estension finie-2. Theorie de Lorenz-Mie generalisee: application a la granulometrie optique," J. Aerosol Sci. 19, 55-64 (1988).

48. Ref. 31, p. 665 . 